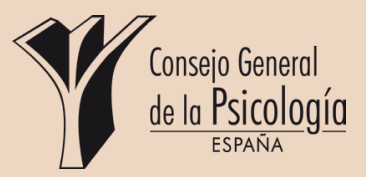

REVISTA IBEROAMERICANA DE

PSICOLOGIA Y SALUD

Revista Oficial de la Federación Iberoamericana de Asociaciones de Psicología (FIAP)

[Official Journal of the Latin-American Federation of Psychological Associations]

\title{
La respuesta placebo en la depresión: Una revisión sistemática
}

\author{
Sonia Pérez-Vela y José Francisco Navarro*
}

Facultad de Psicología, Universidad de Málaga, España.

- Recibido: 29- Junio -2016 - Aceptado: 25- Noviembre -2016

RESUMEN. El objetivo de la presente revisión sistemática es examinar la respuesta placebo en la depresión, recabando información de los estudios publicados desde el año 2000 a la actualidad y plasmando de forma organizada los resultados de los mismos. La búsqueda se ha realizado en las bases de datos: Medline, PILOTS, Psycinfo, Scopus y Pubmed. Esta búsqueda electrónica ha sido complementada con una manual. Con ambas, se han incluido 17 documentos. Los resultados muestran la superioridad de los antidepresivos sobre los placebos; no obstante, la respuesta terapéutica ante los placebos también es elevada en todos los estudios, sobre todo en aquellos que utilizan "placebos activos". Se describen también los factores que modulan a la respuesta placebo, destacando la severidad inicial de la depresión y el número de sesiones de seguimiento. Asimismo, se exponen los hallazgos encontrados a nivel psicobiológico. Por último, se mencionan las limitaciones potenciales, las posibles directrices a seguir en investigaciones futuras y cómo el conocimiento de dicha respuesta podría afectar a la intervención en la práctica clínica.

PALABRAS CLAVE: Respuesta Placebo, Efecto Placebo, Depresión, Revisión sistemática.

\section{Placebo response in depression: A systematic review.}

ABSTRACT. The aim of this systematic review is to examine the placebo response in depression, gathering and organizing studies published from 2000 to present day. The search has been conducted using Medline, PILOTS, Psycinfo, Scopus and Pubmed electronic databases, complemented with a manual one, and it has resulted in a selection of 17 articles. From these studies, the superiority of antidepressants over placebos is evidenced, although there is a high overall response in all cases, particularly when "active placebos" are used. Modulating factors behind placebo responses are also described, with special emphasis on the initial severity of the depression and the number of follow-up assessments. Psychobiology findings are explained as well. As concluding remarks, potential limitations of the employed methodologies, feasible directives for future research, and the influence of the knowledge of placebo responses in clinical practices are presented.

KEYWORDS: Placebo Effect, Placebo Response, Depression, Systematic Review.

\section{- DEFINICIÓN DE PLACEBO}

La historia de la medicina está muy ligada a la historia de los placebos, por ejemplo, la medicina precientífica se basaba en bizarras e inefectivas intervenciones médicas y en la

\footnotetext{
*Correspondencia: José Francisco Navarro.

Facultad de Psicología, Universidad de Málaga.

Código Postal: 29071, Málaga (España).

E-mail: navahuma@uma.es
}

creencia de que estos tratamientos eran efectivos (Benedetti, 2009). Etimológicamente, la palabra placebo deriva del latín "placere" y significa "complacer". Este significado se asociaba a la creencia de que el placebo era dado a los pacientes para contentarlos más que para beneficiarlos (Choliz \& Capafons, 1991).

El término placebo fue usado por primera vez en 1784 para desacreditar al mesmerismo y, a partir de 1787, se incluye en los diccionarios médicos como "medicina falsa" (Kaptchuk, 2001). No obstante, no se le presta mucha atención hasta la llegada de la segunda guerra 
mundial, cuando Henry Beecher, que trabajaba como médico militar, ante la escasez de morfina empezó a inyectarles a los soldados suero salino y observó que muchos de ellos mostraban una rápida mejoría (Kaptchuk, Kerr \& Zanger, 2009).

A su vuelta a los EEUU, en 1955, empezó a realizar ensayos controlados aleatorios (EAC) en los cuales se comparaba la eficacia de un fármaco con un placebo de idéntica apariencia, y a partir de este momento crece el interés por el placebo y sus efectos (Sanchis Aldás, 2012).

Sin embargo, definir el placebo y sus términos relacionados no es tarea fácil, puesto que a menudo se encuentran diferentes acepciones en la literatura científica. En un intento por esclarecer estos términos se presenta un resumen de los mismos (tabla 1). A pesar de estas matizaciones, a menudo términos como "efecto placebo" y "respuesta placebo" se usan de forma intercambiable.

Tabla 1

Definición de placebo y términos relacionados

\begin{tabular}{|c|c|}
\hline Término & Definición \\
\hline Placebo & $\begin{array}{l}\text { Sustancia o procedimiento que no tiene poder inherente para } \\
\text { producir el efecto terapéutico buscado. }\end{array}$ \\
\hline $\begin{array}{l}\text { Efecto } \\
\text { placebo }\end{array}$ & $\begin{array}{c}\text { Cualquier mejoría derivada del hecho de recibir un placebo o } \\
\text { estar bajo un procedimiento determinado. Incluiría otros efectos } \\
\text { como remisión espontánea, regresión a la media, sesgos del } \\
\text { terapeuta o el paciente, habituación o efectos de las co- } \\
\text { intervenciones. }\end{array}$ \\
\hline $\begin{array}{l}\text { Respuesta } \\
\text { placebo }\end{array}$ & $\begin{array}{c}\text { Mejoras, tanto a nivel psicológico como fisiológico, producidas } \\
\text { por el placebo, que se atribuyen a factores psicosociales, por } \\
\text { ejemplo, las expectativas. }\end{array}$ \\
\hline $\begin{array}{l}\text { Placebos } \\
\text { inactivos vs } \\
\text { activos }\end{array}$ & $\begin{array}{l}\text { Los placebos inactivos son sustancias inertes mientras que los } \\
\text { placebos activos no lo son y mimetizan los efectos secundarios } \\
\text { de los tratamientos farmacológicos. }\end{array}$ \\
\hline $\begin{array}{l}\text { Placebos } \\
\text { puros vs } \\
\text { impuros }\end{array}$ & $\begin{array}{l}\text { Los placebos puros no tienen ningún efecto farmacológico, en } \\
\text { cambio los impuros sí los tienen pero no para la condición en la } \\
\text { que están siendo usados (ej. antibióticos en infecciones virales). }\end{array}$ \\
\hline
\end{tabular}

Un aspecto clave para entender el efecto placebo es el contexto psicosocial que rodea al tratamiento, incluyendo elementos como: la actitud del personal médico, el ambiente del hospital, la máquinas usadas, las palabras empleadas por el personal sanitario o el olor, son estímulos sensoriales que pueden influir positivamente $\mathrm{O}$ negativamente sobre la sintomatología (Benedetti, 2014).

\section{- MECANISMOS IMPLICADOS EN LA RESPUESTA PLACEBO}

Al igual que no existe una única respuesta placebo, tampoco existe un único mecanismo de acción de las mismas (Benedetti, 2014). Estos mecanismos han sido examinados por los investigadores desde diferentes puntos vista, tales como el psicológico y el neurobiológico.

1.1) Mecanismos psicológicos: Múltiples mecanismos psicológicos pueden contribuir a la respuesta placebo, entre ellos encontramos: expectativas, condicionamiento, aprendizaje, memoria o motivación (Price, Finniss \& Benedetti, 2008). Se ha constatado que el contexto psicosocial que rodea al paciente actuaría a través de estos mecanismos psicológicos de modo consciente o inconsciente. Cuando actúa a nivel consciente implica complejos mecanismos cognitivos: expectativas, creencia en la eficacia del tratamiento, confianza y esperanza. Mientras que cuando lo hace a través de los mecanismos inconscientes involucra básicamente al condicionamiento clásico (Benedetti, 2014). Por lo tanto, las expectativas y la anticipación de mejora juegan un papel fundamental cuando están involucradas las funciones fisiológicas a nivel consciente, mientras que el condicionamiento clásico será el elemento principal cuando las funciones fisiológicas están influidas a nivel inconsciente (Benedetti et al., 2003).

Las expectativas producirían reajustes cognitivos y promoverían un comportamiento adaptado a la situación. De este modo, las expectativas positivas llevarían a adoptar un comportamiento específico que produciría la mejora (Bandura, 1977). No obstante las expectativas no actúan en solitario, ya que otros factores como la memoria, la motivación o las emociones (Geers, Helfer, Kosbab,Weiland \& Landry, 2005), así como las experiencias previas con otras enfermedades las modularían (Brody, 2000). Además, las expectativas influirían en otros aspectos psicológicos, como las creencias, y a través de ellas en la percepción de control (Benedetti, 2014).

Asimismo, las expectativas actúan sobre mecanismos cerebrales que preparan al cuerpo para el evento, reduciendo la ansiedad y activando las redes neuronales involucradas en los mecanismos de recompensa. El núcleo 
accumbens juega un papel fundamental en el mecanismo de refuerzo mediado por la dopamina; otras áreas implicadas serían el área tegmental ventral, la amígdala, la sustancia gris periacueductal y el hipotálamo. Se ha demostrado en diversos estudios que la activación dopaminérgica está relacionada con la expectativa de recompensa (Fuente-Fernández et al., 2001).

Por otro lado, el condicionamiento clásico se produciría en aquellos casos en los que se hubiese expuesto al sujeto con anterioridad a un fármaco activo y se desarrollaría en tres fases: a) antes del condicionamiento, se administraría un fármaco activo (El) que produciría cambios fisiológicos en diferentes parámetros (RI). En esta fase el EN (placebo) no produciría ningún cambio a nivel fisiológico; b) fase de adquisición, en la cual se producirían repetidas asociaciones entre el El (fármaco activo) seguido del EN (placebo); c) fase de evocación, el EC (placebo) elicitaría cambios fisiológicos (RC) (Doering \& Rief, 2012). Por tanto, los estímulos que acompañaban a los fármacos activos (personas, lugares, píldoras o procedimientos) se convierten en estímulos condicionados con capacidad para evocar, en mayor o menor medida, la reacción fisiológica pertinente (Montgomery \& Kirsch, 1997).

La respuesta placebo es más potente después de un protocolo de condicionamiento (Amanzio \& Benedetti, 1999), induciendo este procedimiento cambios en procesos fisiológicos como la secreción hormonal (Benedetti et al., 2003) y la respuesta inmune (Goebel et al., 2002).

Los mecanismos de las expectativas y del condicionamiento no son excluyentes, se influyen entre sí, modulando mutuamente su acción, y representan dos caras de la misma moneda (Stewart-Williams \& Pood, 2004). Ejercen diferente acción a nivel fisiológico: las expectativas influirán, p.ej., sobre el dolor y el comportamiento motor, mientras que el condicionamiento lo hará sobre la secreción hormonal (Bennedetti et al., 2003). Las expectativas ocurrían en primer lugar y el condicionamiento en segundo lugar; por este motivo, el primer emparejamiento será crítico para el desarrollo de la respuesta placebo: a mayores expectativas, mayor efecto placebo y potencialmente mayor condicionamiento asociado con la toma de ese fármaco (Finnis, Kaptchuk, Miller \& Benedetti, 2010).
1.2) Mecanismos neurobiológicos: Estos mecanismos neurobiológicos han sido analizados principalmente en la analgesia del dolor. En este sentido, numerosos estudios han demostrado que la respuesta placebo está relacionada con los sistemas opioides y no opioides (Colloca \& Benedetti, 2005). En el primer caso, se ha sugerido que la administración del placebo podría acompañarse de un aumento de la secreción de endorfinas. Una prueba a favor de esta hipótesis es que la analgesia placebo es típicamente bloqueada por el antagonista opioide naloxona (Benedetti, 1996), mientras que en el segundo caso no (Amanzio \& Benedetti, 1999). El uso de uno u otro mecanismo depende del procedimiento aplicado para inducir la respuesta placebo.

Otros experimentos que apoyan el papel de los opioides son los que han evaluado a los antagonistas de la colecistokinina (CCK). Dado el efecto antiopioide de la CCK, su antagonista proglumida es capaz de aumentar el efecto analgésico a través de la potenciación de los sistemas opioides activados por el placebo (Benedetti, Amanzio \& Maggi, 1995; Benedetti, 1997). Así, la respuesta placebo analgésica parece resultar de un equilibrio entre opioides endógenos y CCK.

Encuanto a lasáreas cerebrales implicadas, se ha observado que la administración del placebo activa la corteza del cíngulo anterior rostral (CCAr), la corteza orbitofrontal (OrbF) y la ínsula anterior (INSa) (Aguilar, 2006). Además, la evidencia disponible sugiere que un circuito descendente CCAr-sustancia gris periacueductal-puente-bulbo, actúa como modulador del dolor y estaría involucrado en la analgesia del placebo (Harrington, 1997).

Otro aspecto destacable en los experimentos realizados hasta la fecha es que en la fase de anticipación de la respuesta analgésica se halló activación en varias regiones corticales: prefrontal dorsolateral, OrbF, prefrontal medial y anterior, parietal superior y periacueductal, lo cual puede indicar que se activa una red cognitiva evaluativa justo antes de la respuesta placebo (Lieberman, 2004; Wager, 2004), y ya en esta temprana fase la elevada actividad de la sustancia gris periacueductal podría indicar que se está produciendo una liberación de opioides endógenos (Wager, 2005). 


\section{- OTROS FACTORES}

El placebo es un fenómeno complejo en el que además de los mecanismos expuestos anteriormente pueden influir otros factores. Por ejemplo, algunos rasgos de la personalidad. Así se ha encontrado que en las personas con alto grado de sugestionabilidad (De Pascalis, Chiaradia \& Carotenuto, 2002), optimistas (Geers, Wellman, Fowler, Helfer \& France, 2010) o con bajos niveles de ansiedad (Morton, Watson, El-Deredy \& Jones, 2009) la magnitud de la respuesta placebo sería mayor, actuando estos rasgos como predictores de la misma. Asimismo, algunas variantes genéticas (polimorfismos) pueden influir igualmente en la respuesta placebo en determinados trastornos psiquiátricos (Furmark, Appel \& Henningsson, 2008).

\section{- LA RESPUESTA PLACEBO EN LA DEPRESIÓN}

La depresión mayor afecta a aproximadamente 120 millones de personas en el mundo y es la primera causa de discapacidad por enfermedad mental (Kessler, Chiu \& Dember, 2005). Aunque suele existir una clara respuesta a la medicación antidepresiva, la respuesta ante el placebo es casi igual de potente (Kirsch \& Sapirstein, 1998). Es importante destacar que la depresión es el trastorno psicológico donde se ha observado una respuesta mayor al tratamiento con placebo. En los ensayos clínicos con adultos, la respuesta media a la medicación fue del 45$70 \%$, comparada con la respuesta media ante el placebo que fue del 30-50\%; esta respuesta ha aumentado un $7 \%$ por cada década en los últimos 30 años (Mora, Nestorius \& Rief, 2011 ; Rutherford \& Roose, 2012).

Existen datos que indican que los pacientes tienen más posibilidades de recaer en la depresión después de haber estado bajo un tratamiento con antidepresivos que si han estado bajo otras condiciones terapéuticas (incluyendo el placebo) (Andrews, Thomson, Amstadter \& Neale, 2012).

\section{- JUSTIFICACIÓN DE LA NECESIDAD Y RELEVANCIA DEL PRESENTE TRABAJO}

Teniendo en cuenta todo lo anteriormente expuesto, podría ser necesario realizar una revisión sistemática sobre el placebo en la depresión, con el objetivo de proporcionar datos recientes que aporten luz sobre este controvertido tema.

En esta revisión presentamos una síntesis de la información científica disponible, con el fin de esclarecer las posibles controversias al respecto, así como la identificación de áreas de incertidumbre donde sean necesarias investigaciones adicionales, lo cual se considera primordial tanto para desarrollar una práctica clínica basada en la evidencia, como para disponer de una herramienta fundamental en la toma de decisiones sanitarias (Ferreira González, Urrutia \& Alonso-Coello, 2011).

\section{OBJETIVS}

La investigación sobre el placebo sigue siendo escasa y en ocasiones contradictoria. Asimismo, es obvio que la depresión es uno de los principales problemas de salud en la actualidad, afectando a millones de personas y con unos costes a nivel económico muy elevados. Ante ello, el objetivo principal de la presente revisión sistemática es recopilar la información existente sobre el placebo en la depresión desde 2000 hasta la actualidad y facilitar su uso a todo aquel profesional interesado en la temática, plasmando de forma organizada los resultados de los estudios encontrados.

\section{MÉTODO}

\section{- ESTRATEGIA DE BÚSQUEDA UTILIZADA}

La búsqueda de las investigaciones se ha realizado en diferentes bases de datos, a saber: Medline, PILOTS, Psycinfo, Scopus y Pubmed. Se ha restringido la búsqueda a aquellos artículos publicados desde el 2000 hasta la actualidad. Los términos empleados para tal búsqueda han sido: "placeboeffect", "placeboresponse", "depression", "mayor depression disorder" y "bipolar disorder". Específicamente, la combinación de términos introducida fue la siguiente: Title ("placebo effect" OR "placebo response") AND Title (depression OR "mayor depression disorder") NOT Title ("bipolar disorder"). Las búsquedas se llevaron a cabo entre el 10 de noviembre de 2015 y el 15 de marzo de 2016.

Para el desarrollo y redacción de la 
revisión sistemática se han seguido las normas de la declaración PRISMA 2015 (Moher et al., 2015), cuyas siglas significan "Preferred Reporting Items for Systematic Reviews and Meta-Analyses". Se trata de una propuesta para mejorar la publicación de revisiones sistemáticas y metaanálisis consistente en una lista de comprobación estructurada de 27 ítems que sigue la estructura básica de un artículo científico y detalla los contenidos específicos que deberían reportarse en cada sección del mismo.

\section{- CRITERIOS DE INCLUSIÓN}

Los criterios de inclusión de la búsqueda han sido los siguientes:

1 - Que se centraran en el estudio del placebo en la depresión.

2- Que sean estudios experimentales.

3- Que los sujetos contaran únicamente con el diagnóstico de depresión mayor sin otros trastornos.

4- Período temporal de publicación de 2000 en adelante.

Todos los artículos incluidos en la revisión cumplen los anteriores cuatro criterios.

\section{RESULTADOS}

\section{- PROCESO DE SELECCIÓN DE LOS} ARTÍCULOS: DIAGRAMA DE FLUJO

El número de artículos encontrados fue 1.195. Tras el proceso de identificación, se pasó a la selección y, de este total, se eliminaron 684 artículos duplicados; a continuación, evaluando los estudios potenciales respecto a los criterios de inclusión, se eliminaron otros 497 estudios más (330 por título, 132 tras lectura del resumen y 35 tras lectura completa). Por tanto, tras la búsqueda electrónica quedaron 14 estudios válidos. No obstante, dichos resultados han sido ampliados a través de una búsqueda manual a partir de las referencias bibliográficas de los artículos previamente seleccionados. Con este procedimiento, se añadieron 3 artículos válidos más, por lo que, finalmente, el total de artículos incluidos en la revisión ascendió a 17.

A continuación, se muestra el proceso mencionado de identificación, selección, elegibilidad e inclusión de los artículos en un diagrama de flujo.

\section{REVISIÓN SISTEMÁTICA, 2000-2016}
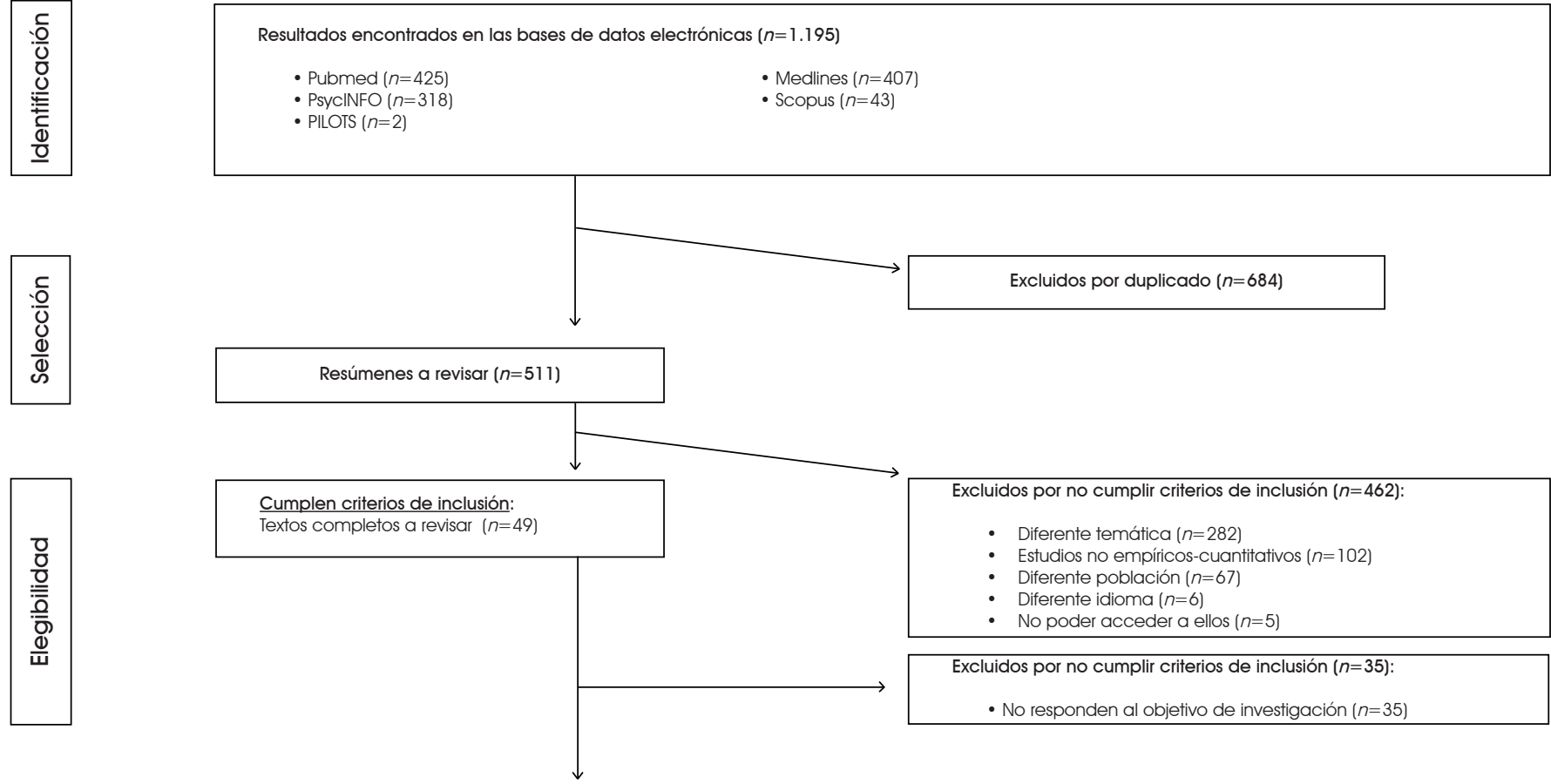

Estudios incluidos de las bases de datos $(n=14)$

Estudios adicionales añadidos a partir de referencias bibliográficas $(n=3)$

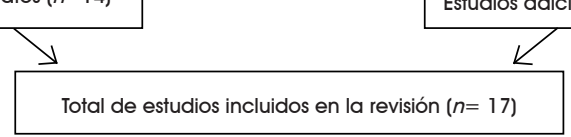




\section{- DESCRIPCIÓN DE LAS CARACTERÍSTICAS DE LOS ESTUDIOS}

La tabla 2 muestra las principales características de los 17 estudios, incluyendo: objetivos, muestra, media de edad, distribución por género, variables psicológicas y físicas y los instrumentos de medida para las mismas.

Tabla 2

Descripción de objetivos, muestra y variables considerables

\begin{tabular}{|c|c|c|c|c|}
\hline AUTORES Y AÑO & $\begin{array}{l}\text { OBJETIVO/S DEL } \\
\text { ESTUDIO }\end{array}$ & $\begin{array}{l}\text { POBLACIÓN Y } \\
\text { PATOLOGÍA }\end{array}$ & $\begin{array}{c}\text { MUESTRA: } N \text {, } \\
\text { EDAD MEDIA }(M) \text {, } \\
\text { DESVIACIÓN TÍPICA } \\
(S D), \% \\
\text { VARONES/MUJERES. }\end{array}$ & $\begin{array}{c}\text { VARIABLES } \\
\text { PSICOLÓGICAS Y } \\
\text { FÍSICAS EVALUADAS E } \\
\text { INSTRUMENTOS }\end{array}$ \\
\hline $\begin{array}{c}\text { Bridge, Birmaher, lyengar, Barbe y } \\
\text { Brent (2009) }\end{array}$ & $\begin{array}{l}\text { Examinar las características } \\
\text { y predictores de la respuesta } \\
\text { placebo en la depresión mayor } \\
\text { pediátrica. }\end{array}$ & $\begin{array}{l}\text { Niños y adolescentes con } \\
\text { diagnóstico de depresión mayor. }\end{array}$ & $\begin{array}{c}12 \text { estudios } \\
N=2,862 \\
M=12.3 \\
S D=3,6 \\
53.14 \% \text { Mujeres } \\
46.86 \% \text { Hombres }\end{array}$ & Depresión (CDRS-R, CGI-S) \\
\hline $\begin{array}{l}\text { *Kirsch, Deacon, Huedo-Medina, } \\
\text { Scoboria, Moore y Johnson (2008) }\end{array}$ & $\begin{array}{c}\text { Establecer la relación entre la } \\
\text { severidad de la depresión y la } \\
\text { eficacia de los antidepresivos. A } \\
\text { la vez que se extraen datos sobre } \\
\text { la respuesta placebo. }\end{array}$ & $\begin{array}{l}\text { Adultos con diagnóstico de } \\
\text { depresión mayor. }\end{array}$ & $\begin{array}{l}35 \text { estudios } \\
N=5,133\end{array}$ & Depresión (HRSD) \\
\hline $\begin{array}{l}\text { *Mancini, Wade, Perugi , Lenox- } \\
\text { Smith y Schacht (2014) }\end{array}$ & $\begin{array}{l}\text { Identificar los parámetros } \\
\text { específicos que dan lugar a } \\
\text { una menor respuesta placebo } \\
\text { y una mayor eficacia de los } \\
\text { antidepresivos, para así poder } \\
\text { mejorar los experimentos que se } \\
\text { realizan con estos fármacos. }\end{array}$ & $\begin{array}{l}\text { Adultos con diagnóstico de } \\
\text { depresión mayor. }\end{array}$ & $\begin{array}{c}14 \text { estudios } \\
\mathrm{N}=4,661 \\
\mathrm{M}=45.9 \\
\mathrm{SD}=15.87 \\
64.9 \% \text { Mujeres } \\
35.1 \% \text { Hombres }\end{array}$ & Depresión (HRSD) \\
\hline $\begin{array}{l}\text { Mayberg, Silva, Brannan, Tekell, } \\
\text { Mahurin, McGinnis y Jerabek } \\
\text { (2002) }\end{array}$ & $\begin{array}{l}\text { Comprobar si la administración } \\
\text { de placebo produce una } \\
\text { respuesta a nivel neuroanatómico } \\
\text { semejante a la observada tras el } \\
\text { tratamiento con antidepresivos. }\end{array}$ & $\begin{array}{l}\text { Adultos con diagnóstico de } \\
\text { depresión mayor (hospitalizados). }\end{array}$ & $\begin{array}{c}N=17 \\
M=49 \\
S D=9 \\
100 \% \text { Hombres }\end{array}$ & $\begin{array}{c}\text { Depresión (HRSD, MINI, Neurocog) } \\
\text { Metabolismo de la glucosa (PET, } \\
\text { FDG) }\end{array}$ \\
\hline Moncrieff, Wessely y Hardy (2004) & $\begin{array}{l}\text { Investigar la eficacia de los } \\
\text { antidepresivos tricíclicos y } \\
\text { compararla con la de los } \\
\text { placebos activos que contienen } \\
\text { atropina. }\end{array}$ & $\begin{array}{l}\text { Adultos con diagnóstico de } \\
\text { depresión mayor. }\end{array}$ & $\begin{array}{c}9 \text { estudios. } \\
N=751 \\
M=43.85 \\
S D=9.2 \\
61.3 \% \text { Mujeres } \\
38.7 \% \text { Hombres }\end{array}$ & $\begin{array}{c}\text { Depresión (HRSD, GCI, PPCPR, } \\
\text { PCDPT, CMS, PSRIP, BDI, MMPI,IMPS, } \\
\text { CGI, TDS) }\end{array}$ \\
\hline $\begin{array}{c}\text { *Leuchter, Cook, Witte, Morgan y } \\
\text { Abrams (2002) }\end{array}$ & $\begin{array}{l}\text { Examinar la actividad cerebral } \\
\text { de los sujetos en dos situaciones } \\
\text { experimentales (medicación } \\
\text { o placebo) y determinar si } \\
\text { mediante QEEG se detectan } \\
\text { diferencias significativas en dicha } \\
\text { actividad entre los sujetos que } \\
\text { responden a la medicación y los } \\
\text { que responden al placebo. }\end{array}$ & $\begin{array}{l}\text { Adultos con diagnóstico de } \\
\text { depresión mayor. }\end{array}$ & $\begin{array}{c}N=51 \\
M=41.45 \\
S D=12 \\
62.75 \% \text { Mujeres } \\
37.25 \% \text { Hombres }\end{array}$ & $\begin{array}{c}\text { Depresión (HRSD) } \\
\text { Actividad cerebral (QEEG) }\end{array}$ \\
\hline $\begin{array}{l}\text { Leuchter, Hunter, Tartter y Cook } \\
\text { (2014) }\end{array}$ & $\begin{array}{l}\text { Examinar los efectos de la } \\
\text { medicación, el placebo y el } \\
\text { "apoyo terapéutico" durante } \\
\text { el desarrollo de un tratamiento, } \\
\text { así como las relaciones entre } \\
\text { las expectativas y la alianza } \\
\text { terapéutica y su contribución a } \\
\text { la mejora de la sintomatología } \\
\text { depresiva. }\end{array}$ & $\begin{array}{l}\text { Adultos con diagnóstico de } \\
\text { depresión mayor. }\end{array}$ & $\begin{array}{c}N=88 \\
M=43 \\
S D=13.51 \\
62.5 \% \text { Mujeres } \\
37.5 \% \text { Hombres }\end{array}$ & $\begin{array}{c}\text { Depresión (HRSD, MINI) } \\
\text { Expectativas (PAEF) } \\
\text { Alianza terapéutica(CALPAS) }\end{array}$ \\
\hline $\begin{array}{l}\text { Peciña, Bohnert, Sikora, Avery, } \\
\text { Langenecker, Mickey y Zubieta } \\
\text { (2015) }\end{array}$ & $\begin{array}{l}\text { Examinar los mecanismos } \\
\text { neuroquímicos que subyacen en } \\
\text { el efecto placebo, explorando } \\
\text { las diferencias entre el } \\
\text { placebo "activo", el placebo } \\
\text { "inactivo" y los antidepresivos. } \\
\text { y comparar diferentes formas } \\
\text { de administración de placebos: } \\
\text { intravenosa y oral. }\end{array}$ & $\begin{array}{l}\text { Adultos con diagnóstico de } \\
\text { depresión mayor. }\end{array}$ & $\begin{array}{c}\mathrm{N}=35 \\
\mathrm{M}=35 \\
\mathrm{SD}=13 \\
65.71 \% \text { Mujeres } \\
34.29 \% \text { Hombres }\end{array}$ & $\begin{array}{l}\text { Depresión (QIDS-SR16, PIDS) } \\
\text { Actividad cerebral (PET) }\end{array}$ \\
\hline
\end{tabular}




\begin{tabular}{|c|c|c|c|c|}
\hline AUTORES Y AÑO & $\begin{array}{c}\text { OBJETIVO/S DEL } \\
\text { ESTUDIO }\end{array}$ & $\begin{array}{l}\text { POBLACIÓN Y } \\
\text { PATOLOGÍA }\end{array}$ & $\begin{array}{l}\text { MUESTRA: } N \text {, } \\
\text { EDAD MEDIA (M), } \\
\text { DESVIACIÓN TÍPICA } \\
\quad(S D), \% \\
\text { VARONES/MUJERES. }\end{array}$ & $\begin{array}{c}\text { VARIABLES } \\
\text { PSICOLÓGICAS Y } \\
\text { FÍSICAS EVALUADAS E } \\
\text { INSTRUMENTOS }\end{array}$ \\
\hline Posternak y Zimmerman (2007) & $\begin{array}{l}\text { Estimar el impacto terapéutico } \\
\text { que producen las sesiones de } \\
\text { seguimiento sobre la respuesta } \\
\text { placebo en los estudios sobre la } \\
\text { eficacia de los antidepresivos. }\end{array}$ & $\begin{array}{l}\text { Adulitos con diagnóstico de } \\
\text { depresión mayor. }\end{array}$ & $\begin{array}{c}41 \text { estudios } \\
\mathrm{N}=7,674\end{array}$ & Depresión (HRSD) \\
\hline $\begin{array}{l}\text { Russowsky, Lopes, Kaptchuck y } \\
\text { Fregni (2009) }\end{array}$ & $\begin{array}{l}\text { Estudiar la respuesta placebo } \\
\text { que se produce en tratamientos } \\
\text { farmacológicos (escitalopram) } \\
\text { y no farmacológicos, en } \\
\text { concreto, en el tratamiento } \\
\text { con estimulación magnética } \\
\text { transcraneal repetitiva. }\end{array}$ & $\begin{array}{l}\text { Adultos con diagnóstico de } \\
\text { depresión mayor. }\end{array}$ & $\begin{array}{c}41 \text { estudios } \\
\mathrm{N}=5,076 \\
\mathrm{M}=45.20 \\
\mathrm{SD}=10.55 \\
60.45 \% \text { Mujeres } \\
39.55 \% \text { Hombres }\end{array}$ & Depresión (HRSD, MADRS) \\
\hline $\begin{array}{l}\text { Rutherford, Mori, Sneed, Pimontel } \\
\text { y Roose (2012) }\end{array}$ & $\begin{array}{l}\text { Calcular el grado en el que la } \\
\text { recuperación espontánea que } \\
\text { se produce en los pacientes } \\
\text { durante la lista de espera de un } \\
\text { tratamiento, afecta a la respuesta } \\
\text { placebo. }\end{array}$ & $\begin{array}{l}\text { Adolescentes y adultos con } \\
\text { diagnóstico de depresión mayor. }\end{array}$ & $\begin{array}{c}10 \text { estudios } \\
\mathrm{N}=340 \\
\mathrm{M}=36.9 \\
\mathrm{SD}=16.4 \\
73 \% \text { Mujeres } \\
26.7 \% \text { Hombres }\end{array}$ & $\begin{array}{l}\text { Depresión (HRSD, GCI, BDI, } \\
\text { MADRS) }\end{array}$ \\
\hline Rutherford, Sneed y Roosse (2009) & $\begin{array}{l}\text { Estudiar si la duración de } \\
\text { tratamiento y usar un grupo } \\
\text { placebo como control afectan } \\
\text { al resultado de los ensayos con } \\
\text { antidepresivos. }\end{array}$ & $\begin{array}{l}\text { Adultos con diagnóstico de } \\
\text { depresión mayor. }\end{array}$ & $\begin{array}{l}90 \text { estudios } \\
\mathrm{N}=16,545\end{array}$ & $\begin{array}{l}\text { Depresión (HRSD, MADRS, BDI, } \\
\text { CGI) }\end{array}$ \\
\hline $\begin{array}{l}\text { Rutherford, Wall, Glass y Stewart } \\
\qquad(2015)\end{array}$ & $\begin{array}{l}\text { Determinar si las expectativas } \\
\text { influyen en el efecto placebo y } \\
\text { nocebo en dos ensayos clínicos. }\end{array}$ & $\begin{array}{l}\text { Adultos con diagnóstico de } \\
\text { depresión mayor. }\end{array}$ & $\begin{array}{c}2 \text { estudios } \\
\mathrm{N}=664 \\
\mathrm{M}=39.47 \\
\mathrm{SD}=10.77 \\
64.91 \% \text { Mujeres } \\
35.09 \% \text { Hombres }\end{array}$ & $\begin{array}{c}\text { Depresión (HRSD,CGI) } \\
\text { Expectativas (PAEF) }\end{array}$ \\
\hline $\begin{array}{l}\text { Sikora, Heffernan, Avery, Mickey, } \\
\text { Zubieta y Peciña (2016) }\end{array}$ & $\begin{array}{l}\text { Investigar si existe una relación } \\
\text { entre la conectividad funcional } \\
\text { en estado de reposo y los } \\
\text { efectos de los antidepresivos y el } \\
\text { placebo, es decir, si estas redes } \\
\text { pueden predecir o modular la } \\
\text { respuesta ante los fórmacos y el } \\
\text { placebo. }\end{array}$ & $\begin{array}{l}\text { Adultos con diagnóstico de } \\
\text { depresión mayor. }\end{array}$ & $\begin{array}{c}\mathrm{N}=29 \\
\mathrm{M}=32 \\
\mathrm{SD}=13 \\
72.41 \% \text { Mujeres } \\
27.59 \% \text { Hombres }\end{array}$ & $\begin{array}{c}\text { Depresión (QIDS-16SR, HRSD, } \\
\text { MADRS) } \\
\text { Actividad cerebral (fRMN) }\end{array}$ \\
\hline Undurraga y Baldessarini (2012) & $\begin{array}{l}\text { 1. Comparar la eficacia de } \\
\text { los antidepresivos con la del } \\
\text { placebo. } \\
\text { 2. Comparar la eficacia de los } \\
\text { antidepresivos clásicos frente a } \\
\text { los modernos. Examinar los factores que } \\
\text { se asocian al declive de la } \\
\text { diferencia entre placebo y } \\
\text { antidepresivos a través de los } \\
\text { años. }\end{array}$ & $\begin{array}{l}\text { Adultos con diagnóstico de } \\
\text { depresión mayor. }\end{array}$ & $\begin{array}{c}122 \text { estudios } \\
\mathrm{N}=27,127 \\
\mathrm{M}=40 \\
\mathrm{SD}=13.54 \\
62 \% \text { Mujeres } \\
38 \% \text { Hombres }\end{array}$ & Depresión (HRSD, MADRS, CGI). \\
\hline $\begin{array}{l}\text { Walsh, Seidman, Sysko y Gould } \\
\text { (2002). }\end{array}$ & $\begin{array}{l}\text { Determinar si las características } \\
\text { y el porcentaje de mejora en el } \\
\text { grupo placebo cambian en los } \\
\text { experimentos con antidepresivos } \\
\text { a través del tiempo. }\end{array}$ & $\begin{array}{l}\text { Adultos con diagnóstico de } \\
\text { depresión mayor. }\end{array}$ & $\begin{array}{l}75 \text { estudios } \\
\mathrm{N}=11,456 \\
\mathrm{M}=42.6 \\
\mathrm{SD}=7.3 \\
60.1 \% \text { Mujeres } \\
39.9 \% \text { Hombres }\end{array}$ & $\begin{array}{l}\text { Depresión (HRSD, MINI) } \\
\text { Expectativas (PAEF) } \\
\text { Alianza terapéutica(CALPAS) }\end{array}$ \\
\hline $\begin{array}{l}\text { Peciña, Bohnert, Sikora, Avery, } \\
\text { Langenecker, Mickey y Zubieta } \\
\text { (2015) }\end{array}$ & $\begin{array}{l}\text { Examinar los mecanismos } \\
\text { neuroquímicos que subyacen en } \\
\text { el efecto placebo, explorando } \\
\text { las diferencias, entre el } \\
\text { placebo "activo", el placebo } \\
\text { "inactivo" y los antidepresivos. } \\
\text { y comparar diferentes formas } \\
\text { de administración de placebos: } \\
\text { intravenosa y oral. }\end{array}$ & $\begin{array}{l}\text { Adultos con diagnóstico de } \\
\text { depresión mayor. }\end{array}$ & $\begin{array}{c}\mathrm{N}=35 \\
\mathrm{M}=35 \\
\mathrm{SD}=13 \\
65.71 \% \text { Mujeres } \\
34.29 \% \text { Hombres }\end{array}$ & Depresión (HRSD) \\
\hline \multicolumn{5}{|c|}{ *Artículos encontrados a partir de la búsqueda manual. } \\
\hline \multicolumn{5}{|c|}{$\begin{array}{l}\text { Nota. FDG = Fluorodexyglucose; HRSD = Hamilton Rating Scale for Depression; PAEF = Patient Attitudes and Expectations Form; CALPAS= California } \\
\text { Pharmacotherapy Alliance Scale; MINI= Mini-International Neuropsychiatric Interview; MADRS= Montgomery- } \text { sberg Depression Rating Scale; GCI= } \\
\text { Global Clinical Improvement; PPCPR= Philadelphia Psychiatric Centre Psychatric Rating Scale; PCDPT= Philadelphia Psychiatric Center Depression } \\
\text { Progress Test; CMS= Clyde Mood Scale; PSRIP= Patient Self Report Inventory of Psychic and Somatic Complaints; BDI = Beck Depression Inventory; } \\
\text { MMPI= Minnesota Multiphasic Personality Inventory; IMPS= Inpatient Multidimensional Psychiatric Scale; CGI= Clinical Global Improvement Scale; } \\
\text { CGI-S:=Clinical Global Improvement Severity Scale; TDS= Total Distress Scale; QIDS-16SR= Quick Inventory of Depression Symptomatology; PIDS= } \\
\text { Patient's Impression of Depression Severity; PET= Positron Emission Tomography; CDRS-R= Children's Depression Rating Scale-Revised; fRMI = Func- } \\
\text { tional magnetic resonance imaging. }\end{array}$} \\
\hline
\end{tabular}


Todos los estudios fueron publicados entre el 2000 y el 2016 . El lugar de realización de la mayor parte de los trabajos fue EE.UU., excepto dos: uno se desarrolló en Francia (Nauret et al., 2013) y otro en Reino Unido (Posternak \& Zimmerman, 2007). Por otro lado, 11 de los estudios fueron metaanálisis y 6 estudios originales. Los diseños usados en la mayoría de los experimentos fueron: el "doble ciego" y los diseños cruzados. La muestra de participantes incluyó desde 17 participantes (Mayberg et al., 2002) hasta 27.127 (Undurraga \& Baldessarini, 2012). Los sujetos fueron elegidos a través de hospitales, colegios u otros centros de la comunidad.

El ratio medio de edad de la mayoría de los estudios estaba comprendido entre los 18 y los 60 años, con una media de 41,48 años, excepto un estudio (Bridge, Birmaher, lyengar, Barbe \& Brent, 2009) cuya población diana eran los niños y adolescentes, siendo la media de edad 12,3 años. Las mujeres contaban con un mayor porcentaje de participación en todos los estudios, excepto en uno (Mayberg et al., 2002) en el cual todos eran hombres.
Además, en este trabajo todos los pacientes estaban hospitalizados, a diferencia de la mayoría de los otros estudios donde seguían tratamiento de modo ambulatorio.

Variables psicológicas estudiadas: la depresión fue medida en los 17 artículos, principalmente mediante la escala para depresión de Hamilton (HRSD) que se utilizó en 15 estudios. También se midieron otras variables como las expectativas (Leuchter, Hunter, Tartter \& Cook, 2014; Rutherford, Wall, Glass \& Stewart, 2015) y la alianza terapeútica (Leuchter, Hunter, Tartter \& Cook, 2014).

Variables físicas estudiadas: Metabolismo de la glucosa (Mayberg et. al., 2002) y actividad cerebral (Peciña et. al., 2015: Sikora et. al., 2016; Leuchter, Cook, Witte, Morgan y Abrams, 2002).

\section{- DESCRIPCIÓN DE LOS PRINCIPALES RESULTADOS HALLADOS}

La tabla 3 muestra los principales resultados de los artículos analizados.

\begin{tabular}{|c|c|c|c|c|}
\hline AUTORES Y AÑO & $\begin{array}{l}\text { DISEÑO DEL } \\
\text { ESTUDIO }\end{array}$ & $\begin{array}{c}\text { ANÁLISIS } \\
\text { ESTADÍSTICO }\end{array}$ & BREVE RESUMEN DE RESULTADOS & CONCLUSIONES \\
\hline $\begin{array}{l}\text { Bridge, Birmaher, lyengar, } \\
\text { Barbe y Brent (2009). }\end{array}$ & Metaanálisis & $\begin{array}{l}\text { Correlación de Pearson } \\
\text { Regresión lineal múltiple } \\
\text { Coeficiente de } \\
\text { correlación de } \\
\text { Spearman } \\
\text { Chi cuadrado }\end{array}$ & $\begin{array}{l}\text { La proporción media de participantes que } \\
\text { respondieron al placebo fue de un } 46 \% \text { frente } \\
\text { al } 59 \% \text { que respondieron al fórmaco. Los más } \\
\text { jóvenes mostraban una respuesta placebo } \\
\text { superior a los más mayores. La proporción de } \\
\text { pacientes que responden al placebo correlaciona } \\
\text { significativamente con el año de publicación, } \\
\text { indicando un incremento de respuesta con el paso } \\
\text { de los años. }\end{array}$ & $\begin{array}{l}\text { La edad y la severidad de la depresión } \\
\text { modulaban la respuesta placebo. La } \\
\text { proporción de pacientes que responden al } \\
\text { placebo se ha incrementado en los últimos } \\
\text { años. }\end{array}$ \\
\hline *Kirsch et al., (2008) & Metaanálisis & $\begin{array}{l}d \text { de Cohen } \\
\text { ANOVA }\end{array}$ & $\begin{array}{l}\text { La media de mejora en el grupo con antidepresivos } \\
\text { fue de } 9.60 \text { en la HRSD, frente a } 7.80 \text { en el grupo } \\
\text { placebo. Venlafaxina y paroxetina fueron los } \\
\text { fármacos que más eficacia mostraron. También } \\
\text { se encontró una correlación negativa entre la } \\
\text { severidad de la depresión y la respuesta placebo, } \\
\text { siendo esta relación mayor en los estudios con } \\
\text { muestras pequeñas. }\end{array}$ & $\begin{array}{l}\text { La diferencia entre los antidepresivos y el } \\
\text { placebo se incrementa según aumenta la } \\
\text { severidad del trastorno, si bien en términos } \\
\text { totales sigue siendo pequeña aunque los } \\
\text { sujetos estuviesen muy depresivos. Está } \\
\text { diferencia es atribuida a una disminución } \\
\text { de la respuesta ante el placebo y no a un } \\
\text { incremento de respuesta ante la medicación. }\end{array}$ \\
\hline $\begin{array}{l}\text { *Mancini, } \\
\text { Lenox-Smith y y } \begin{array}{l}\text { yerugi, } \\
\text { (2014) }\end{array}\end{array}$ & Metaanálisis & ANCOVA & $\begin{array}{l}\text { El tamaño del efecto global (ES) entre la duloxetina } \\
\text { y el placebo fue de }-0.272 \text {, tomando la HRSD } \\
\text { como instrumento de medida. Aunque no hay } \\
\text { interacciones significativas, se encontraron que } \\
\text { los siguientes factores afectan al tamaño global } \\
\text { del efecto: La leve sintomatología inicial de la } \\
\text { depresión afectaba positivamente al efecto } \\
\text { placebo, al igual que la corta duración de los } \\
\text { experimentos, una no adecuada aleatorización y } \\
\text { un } n^{\circ} \text { de evaluaciones de seguimiento mayor. }\end{array}$ & $\begin{array}{l}\text { Los diseños de ensayos simples, con } \\
\text { evaluaciones de seguimiento limitadas, un } \\
\text { tamaño medio de sujetos y una puntuciánón } \\
\text { elevada de severidad del trastorno, así como } \\
\text { sin grupo de control activo podrían conducir } \\
\text { a una mejor detección de la eficacia de los } \\
\text { antidepresivos. }\end{array}$ \\
\hline
\end{tabular}




\begin{tabular}{|c|c|c|c|c|}
\hline AUTORES Y AÑO & $\begin{array}{l}\text { DISEÑO DEL } \\
\text { ESTUDIO }\end{array}$ & $\begin{array}{c}\text { ANÁLISIS } \\
\text { ESTADÍSTICO }\end{array}$ & BREVE RESUMEN DE RESULTADOS & CONCLUSIONES \\
\hline Mayberg et al., (2002) & $\begin{array}{l}\text { Experimental } \\
\text { Doble ciego }\end{array}$ & ANOVA & $\begin{array}{l}\text { Se produjo una mejora en la sintomatología } \\
\text { depresiva en ambos grupos, no hallándose } \\
\text { diferencias significativas en las puntuaciones de las } \\
\text { escalas entre ellos. Los sujetos que respondieron al } \\
\text { placebo mostraron un aumento en el metabolismo } \\
\text { de la glucosa en las siguientes áreas: corteza } \\
\text { prefrontal, premotora y parietal inferior, ínsula } \\
\text { posterior y área cingulada posterior, con una } \\
\text { disminución en el metabolismo enárea subgenual, } \\
\text { hipotálamo, tálamo, ínsula suplementaria sensorial } \\
\text { y parahipocampo. Los sujetos que respondieron a } \\
\text { la medicación mostraron cambios parecidos a los } \\
\text { anteriores, pero además exhibieron un incremento } \\
\text { en el metabolismo del tronco cerebral y un } \\
\text { decremento en el estriado, hipocampo e ínsula } \\
\text { anterior. }\end{array}$ & $\begin{array}{l}\text { La respuesta placebo se asocia a cambios } \\
\text { específicos en la función cerebral. Mientras } \\
\text { que la respuesta a la medicación, además } \\
\text { de presentar estos cambios observados en el } \\
\text { placebo en mayor intensidad, muestran otros } \\
\text { específicos y únicos en el núcleo estriado, el } \\
\text { hipocampo y el tronco encefálico. A destacar } \\
\text { que estos cambios solo se producen en los } \\
\text { sujetos que responden a la medicación y no en } \\
\text { los sujetos medicados pero que no respondieron. } \\
\text { Los resultados de este estudio sugieren que la } \\
\text { adaptación recíproca entre los sistemas límbico } \\
\text { y cortical es necesaria para la disminución de } \\
\text { la depresión, independientemente del tipo de } \\
\text { tratamiento seguido. }\end{array}$ \\
\hline $\begin{array}{l}\text { Moncrieff, Wessely y Hardy } \\
\qquad(2004)\end{array}$ & Metaanálisis & $\begin{array}{c}\text { ANOVA } \\
\text { Chi cuadrado }\end{array}$ & $\begin{array}{l}\text { Se obtuvieron resultados significativos y consistentes } \\
\text { a favor de los antidepresivos, aunque en un } \\
\text { segundo análisis más profundo la diferencia entre } \\
\text { el placebo y el fármaco fue pequeña. }\end{array}$ & $\begin{array}{l}\text { Las diferencias entre los placebos activos y los } \\
\text { antidepresivos fueron pequeñas. Los efectos de } \\
\text { los antidepresivos pueden estar sobreestimados } \\
\text { y los de los placebos subestimados. Limitación: } \\
\text { en algunos estudios los pacientes recibían } \\
\text { terapia cognitiva-conductual conjuntamente } \\
\text { con los medicamentos y este hecho no se } \\
\text { controló. }\end{array}$ \\
\hline Naudet et. al.,(2013) & Metaanálisis & $\begin{array}{c}\text { ANOVA } \\
\text { Chi cuadrado }\end{array}$ & $\begin{array}{l}\text { Los placebos en las } 3 \text { situaciones experimentales } \\
\text { (fluoxetina, venlafaxina y combinación) no } \\
\text { obtuvieron resultados significativos en términos de } \\
\text { respuesta o remisión. Los antidepresivos fueron más } \\
\text { eficaces que los placebos y la venlafaxina más que } \\
\text { la fluoxetina. }\end{array}$ & $\begin{array}{l}\text { La composición exacta del placebo no es } \\
\text { relevante, todos estaban hechos a partir de } \\
\text { sacarosa, aunque con pequeñas variaciones, } \\
\text { esto no repercutió en la respuesta ante } \\
\text { él y obtuvo semejantes respuestas. Los } \\
\text { antidepresivos fueron más eficaces que el } \\
\text { placebo. }\end{array}$ \\
\hline $\begin{array}{l}\text { *Leuchter, Cook, Witte, } \\
\text { Morgan y Abrams (2002) }\end{array}$ & $\begin{array}{l}\text { Experimental } \\
\text { Doble ciego }\end{array}$ & ANOVA & $\begin{array}{l}\text { El } 52 \% \text { de los sujetos que recibieron antidepresivos } \\
\text { respondieron al tratamiento, frente al } 38 \% \text { de los } \\
\text { que recibieron placebo. En los que respondieron al } \\
\text { placebo se observa un incremento de la actividad } \\
\text { prefrontal tras la } 4^{\mathrm{a}} \text { semana de tratamiento que se } \\
\text { convierte en estadísticamente significativo en la } 8^{a} \\
\text { semana. Mientras que en los sujeto que responden } \\
\text { a los antidepresivos se observa un decremento de } \\
\text { la actividad prefrontal, sobre todo en la } 2^{\mathrm{a}} \text { semana. } \\
\text { No se encontraron diferencias significativas en los } \\
\text { resultados finales de mejora en la sintomatología } \\
\text { depresiva entre los dos grupos que respondieron. }\end{array}$ & $\begin{array}{l}\text { El tratamiento con placebo induce cambios en } \\
\text { la función cerebral que son distintos de los que } \\
\text { se producen con la medicación antidepresiva. } \\
\text { Altos niveles de metabolismo pueden estar } \\
\text { asociados a la respuesta placebo y presentar } \\
\text { un patrón único en el área prefrontal. Este } \\
\text { estudio plantea que la respuesta placebo } \\
\text { y la respuesta antidepresiva podrían estar } \\
\text { asociadas a mecanismos de acción diferentes. }\end{array}$ \\
\hline $\begin{array}{c}\text { Leuchter, Hanter, Tartter y } \\
\text { Cook (2014) }\end{array}$ & $\begin{array}{l}\text { Experimental } \\
\text { Doble ciego }\end{array}$ & $\begin{array}{l}\text { ANOVA } \\
t \text { de Student } \\
\text { Regresión lineal } \\
\text { Chi cuadrado } \\
\text { Test de Tukey }\end{array}$ & $\begin{array}{l}\text { Los pacientes asignados al grupo de medicación o } \\
\text { al grupo placebo tuvieron una mejora significativa } \\
\text { de los síntomas depresivos, mientras que en el que } \\
\text { solo recibía "apoyo terapéutico" esto no pasaba. } \\
\text { Se halló una diferencia numérica, aunque no } \\
\text { significativa, a favor del grupo de medicación } \\
\text { versus grupo placebo. Los sujetos que solo recibían } \\
\text { apoyo permanecieron en el tratamiento menos } \\
\text { tiempo que aquellos que tomaban pastillas. } \\
\text { No hubo diferencias entre los distintos tipos de } \\
\text { fármacos (venlafaxina, escitalopram). }\end{array}$ & $\begin{array}{l}\text { Las expectativas pueden representar un } \\
\text { constructo que afecte a la probabilidad con } \\
\text { la que se produce el efecto placebo, sin } \\
\text { embargo, no guarda relación con la alianza } \\
\text { terapéutica ni con la respuesta inicial del } \\
\text { tratamiento. La alianza terapéutica es un fuerte } \\
\text { predictor de la respuesta ante la medicación } \\
\text { y el placebo, hallándose en ambos caso } \\
\text { una relación significativa. El hecho de que el } \\
\text { "apoyo terapéutico" obtenga pobres resultados } \\
\text { en solitario puede indicar cómo los sujetos } \\
\text { atribuyen más expectativas de mejora a las } \\
\text { pastillas. }\end{array}$ \\
\hline $\begin{array}{l}\text { Peciña, Bohnert, Sikora, } \\
\text { Avery, Langenecker, } \\
\text { Mickey y Zubieta (2015) }\end{array}$ & $\begin{array}{l}\text { Experimental } \\
\text { Doble ciego }\end{array}$ & $\begin{array}{l}\text { ANOVA } \\
\text { Chi cuadrado } \\
\text { Coeficiente de } \\
\text { determinación } \\
t \text { de Student }\end{array}$ & $\begin{array}{l}\text { En todos grupos hubo una mejora de la } \\
\text { sintomatología depresiva. El placebo "activo" } \\
\text { obtuvo diferencias con el placebo "inactivo" tras } \\
\text { la primera semana. Además, quienes recibieron } \\
\text { el placebo "activo" en primer lugar obtuvieron } \\
\text { diferencias mayores que los que recibieron } \\
\text { en segundo lugar. Las mujeres mostraron más } \\
\text { reducción de la sintomatología tras la toma de } \\
\text { placebo oral que los hombres. La activación del } \\
\text { núcleo accumbens está asociada a una mejora } \\
\text { de la respuesta del tratamiento antidepresivo. Se } \\
\text { produjo la activación del receptor } \mu \text {-opioide en } \\
\text { múltiples áreas cerebrales tras la administración del } \\
\text { placebo. La remisión fue más notable en los sujetos } \\
\text { que respondieron al placebo (60\%) que aquellos } \\
\text { que no lo hicieron. }\end{array}$ & $\begin{array}{l}\text { El placebo induce la activación del sistema } \\
\mu \text {-opioide en el núcleo accumbens, que está } \\
\text { implicado en el desarrollo de la respuesta } \\
\text { antidepresiva, así como en la respuesta de } \\
\text { resiliencia a la enfermedad. Se cree que el } \\
\text { sistema opioide podría actuar modulando } \\
\text { la sintomatología depresiva. Esta activación } \\
\text { también se da en otras situaciones como el } \\
\text { "dolor" por lo que puede sugerir que existe } \\
\text { un mecanismo neurobiológico del placebo } \\
\text { común en distintas patologías. En la práctica } \\
\text { clínica, la respuesta al placebo podría } \\
\text { indicar la probabilidad del grado de mejora } \\
\text { del paciente, interactuando con el entorno } \\
\text { psicosocial y las aproximaciones cognitivas. }\end{array}$ \\
\hline
\end{tabular}




\begin{tabular}{|c|c|c|c|c|}
\hline AUTORES Y AÑO & $\begin{array}{l}\text { DISEÑO DEL } \\
\text { ESTUDIO }\end{array}$ & $\begin{array}{c}\text { ANÁLISIS } \\
\text { ESTADÍSTICO }\end{array}$ & BREVE RESUMEN DE RESULTADOS & CONCLUSIONES \\
\hline $\begin{array}{l}\text { Posternak y Zimmerman } \\
\text { (2007) }\end{array}$ & Metaanálisis & $\begin{array}{l}\text { Estudios de } \\
\text { cohortes. } \\
\text { Diferencias } \\
\text { de medias } \\
\text { estandarizadas } \\
\text { (DME) }\end{array}$ & $\begin{array}{l}\text { Una sesión extra de seguimiento a las } 3 \text { semanas } \\
\text { estuvo asociada a una reducción de } 0.86 \text { en la } \\
\text { HRSD, mientras que si se producía en la semana } \\
5^{a} \text { el efecto era de } 0.67 \text {. Estos efectos representan } \\
\text { del } 34-44 \% \text { de la respuesta placebo que ocurre } \\
\text { en este marco temporal. Dos sesiones más de } \\
\text { seguimiento se asociaron al doble de reducción } \\
\text { que si se producía solo una, por lo que se sugiere } \\
\text { que el impacto terapéutico es acumulativo y } \\
\text { proporcional. Esta mejora también se producía } \\
\text { en los pacientes que recibían medicación activa } \\
\text { aunque en menor medida. }\end{array}$ & $\begin{array}{l}\text { Las sesiones de evaluación de seguimiento } \\
\text { influyen de forma significativa en el efecto } \\
\text { terapéutico de los participantes que están en } \\
\text { el grupo placebo, representando el } 40 \% \text { de } \\
\text { dicha respuesta placebo. Esto sugiere que la } \\
\text { respuesta placebo depende en gran medida } \\
\text { de la atención y el cuidado recibido durante el } \\
\text { curso de los ensayos clínicos. }\end{array}$ \\
\hline $\begin{array}{l}\text { Russowsky, Lopes, } \\
\text { Kaptchuck y Fregni (2009) }\end{array}$ & Metaanálisis & $\begin{array}{l}\text { d de Cohen } \\
\text { Chi cuadrado } \\
\text { Metaregresión } \\
\text { Modelo de efectos } \\
\text { aleatorios }\end{array}$ & $\begin{array}{l}\text { En todos los estudios se produjo un tamaño del } \\
\text { efecto grande en el grupo placebo, pero fue } \\
\text { mayor en los estudios farmacológicos que en los de } \\
\text { estimulación magnética transcraneal (rTMS). } \\
\text { Los sujetos con depresión severa y refractaria } \\
\text { a tratamiento presentaron una baja respuesta } \\
\text { al placebo. En ninguna de las otras variables } \\
\text { estudiadas edad, género) se obtuvo resultados } \\
\text { significativos. }\end{array}$ & $\begin{array}{l}\text { Puede que el hecho de tomar una píldora } \\
\text { forme parte de un ritual médico más asentado } \\
\text { en nuestra cultura y sobre lo que los pacientes } \\
\text { tengan más expectativas de mejora que el } \\
\text { rTMS, lo cual explique esta diferencia en favor } \\
\text { de los placebos fármacos. Los pacientes que } \\
\text { presentan depresión refractaria no parecen } \\
\text { responder a ningún tipo de tratamiento ya sea } \\
\text { placebo, farmacológico o rTMS. }\end{array}$ \\
\hline $\begin{array}{l}\text { Rutherford, Mori, Sneed, } \\
\text { Pimontel y Rose (2012) }\end{array}$ & Metaanálisis & $\begin{array}{l}\text { d de Cohen } \\
\text { Coeficiente de } \\
\text { determinación } \\
\text { Método de } \\
\text { DerSimonian y Laird }\end{array}$ & $\begin{array}{l}\text { El tamaño del efecto durante la lista de espera fue } \\
0.050 \text {, representando una media de mejora de } 4 \\
\text { puntos en la HRSD. Ésta representa el } 30 \% \text { de la } \\
\text { mejora que se obtiene con la medicación y el } 40 \% \\
\text { que se observa con la administración de placebo. }\end{array}$ & $\begin{array}{l}\text { Los pacientes depresivos mejoran de } \\
\text { forma sustancial incluso sin tratamiento y la } \\
\text { recuperación espontánea representa una } \\
\text { mejora distinta a la observada en la mayoría } \\
\text { de los estudios sobre la respuesta placebo y } \\
\text { debe ser tenida en cuenta en futuros estudios. } \\
\text { No obstante, es poco probable que represente } \\
\text { la magnitud total del efecto placebo que se } \\
\text { produce en los estudios con antidepresivos. }\end{array}$ \\
\hline $\begin{array}{l}\text { Rutherford, Sneed y Roosse } \\
\qquad(2009)\end{array}$ & Metaanálisis & $\begin{array}{l}t \text { de Student } \\
\text { Chi cuadrado }\end{array}$ & $\begin{array}{c}\text { En los estudios que utilizaban un placebo en el } \\
\text { grupo control, la respuesta media a la medicación } \\
\text { fue de un } 52 \% \text { y la de remisión de } 39,7 \% \text {. Mientras } \\
\text { que la respuesta media ante el placebo fue de un } \\
34,7 \% \text { y la remisión de un } 24,5 \% \text {. Por otra parte, en } \\
\text { los estudios con comparator trial la respuesta media } \\
\text { a la medicación fue de un } 65,2 \% \text { y la de la remisión } \\
\text { de un } 48,4 \% \text {. }\end{array}$ & $\begin{array}{l}\text { El descubrimiento de que la respuesta y la } \\
\text { remisión son diferentes significativamente } \\
\text { cuando son administrados en un grupo control } \\
\text { que utiliza el placebo o en uno que utiliza un } \\
\text { "comparator trial". El diseño del estudio afecta } \\
\text { a la velocidad con la que se responde al } \\
\text { tratamiento. Aunque entre la semana 8-12 no } \\
\text { hay diferencias, si en la semana } 12 \text { en la cual } \\
\text { los estudios con más pacientes respondieron } \\
\text { más lentamente. Las altas expectativas de } \\
\text { mejora entre los individuos que participaban } \\
\text { en los "comparator trial", pudieron producir } \\
\text { las diferencias observadas en el desarrollo de } \\
\text { los tratamientos. Estas expectativas pueden } \\
\text { estar relacionadas con una gran alianza } \\
\text { terapéutica, o el seguimiento del tratamiento } \\
\text { en momentos de empeoramiento sintomático o } \\
\text { la percepción de una sintomatología más leve. }\end{array}$ \\
\hline $\begin{array}{c}\text { Rutherford, Wall, Glass y } \\
\text { Stewart (2015) }\end{array}$ & Cruzado & $\begin{array}{l}\text { Modelo de efectos } \\
\text { mixtos longitudinales } \\
\text { Diferencias } \\
\text { de medias } \\
\text { estandarizadas } \\
\text { (DME) } \\
\text { tde Student }\end{array}$ & $\begin{array}{l}\text { La interrupción del tratamiento con fluoxetina se } \\
\text { asoció con un incremento en el riesgo de recaída } \\
\text { en ambos estudios. En el estudio } 1 \text {, el } 42 \% \text { de los } \\
\text { sujetos tratados con fluoxetina frente al } 19 \% \text { del } \\
\text { grupo placebo, continuaron en remisión en la } \\
\text { semana } 62 \text {, mientras que en el segundo estudio } \\
\text { fueron el } 52 \% \text { de los tratados con fluoxetina frente } \\
\text { al } 28 \% \text { tratado con placebo. }\end{array}$ & $\begin{array}{l}\text { Las expectativas de los pacientes influyen } \\
\text { en que los síntomas aumenten o desciendan } \\
\text { inmediatamente después del cambio de } \\
\text { tratamiento y el conocimiento de su situación } \\
\text { experimental (incluso cuando siguen tomando } \\
\text { medicación). La posibilidad de recibir } \\
\text { placebo fue asociada con un empeoramiento } \\
\text { de los síntomas en los dos estudios. El } \\
\text { empeoramiento de la sintomatología estuvo } \\
\text { inversamente asociado con el grado de mejora } \\
\text { experimentado en las primeras semanas de } \\
\text { tratamiento. Estos resultados sugieren que } \\
\text { los cambios en el tratamiento influyen en las } \\
\text { expectativas de mejora de los pacientes, lo } \\
\text { que a su vez afecta a los síntomas depresivos. }\end{array}$ \\
\hline $\begin{array}{l}\text { Sikora, Heffernan, Avery, } \\
\text { Mickey, Zubieta y Peciña } \\
\text { (2016) }\end{array}$ & Cruzado & $\begin{array}{l}\text { ANOVA } \\
\text { Pearson } \\
\text { Coeficiente de } \\
\text { determinación } \\
\text { Regresión lineal }\end{array}$ & $\begin{array}{l}\text { Las reducciones después de una semana con } \\
\text { el placebo "activo" fueron superiores que con } \\
\text { el "inactivo". Los sujetos que tenían una elevada } \\
\text { conectividad funcional en estado de reposo en } \\
\text { la corteza cingulada anterior, previa al inicio del } \\
\text { experimento, desarrollaban una gran respuesta } \\
\text { ante el placebo. El placebo inducía reducciones } \\
\text { de esta actividad, en concreto, de la activación de } \\
\text { la "red de saliencia" (SN) en la corteza cingulada } \\
\text { anterior, que se relaciona con la disminución de } \\
\text { los síntomas depresivos, esta conectividad podría } \\
\text { explicar el } 65 \% \text { de la varianza atribuida al placebo. } \\
\text { Esta relación también se observa tras la toma de } \\
\text { fármacos, aunque después de las } 10 \text { semanas. }\end{array}$ & $\begin{array}{l}\text { Este estudio demuestra que la línea base de la } \\
\text { conectividad funcional en estado de reposo } \\
\text { con la que parte los sujetos en la corteza } \\
\text { cingulada anterior es clave para predecir } \\
\text { su posterior respuesta ante el placebo. Esta } \\
\text { información podría ser útil para identificar } \\
\text { a pacientes que podrían beneficiarse de } \\
\text { tratamientos con dosis bajas de antidepresivos } \\
\text { u otras aproximaciones no farmacológicas, } \\
\text { abriendo también una futura línea de } \\
\text { investigación sobre los biomarcadores en los } \\
\text { ensayos clínicos sobre efecto placebo. }\end{array}$ \\
\hline
\end{tabular}




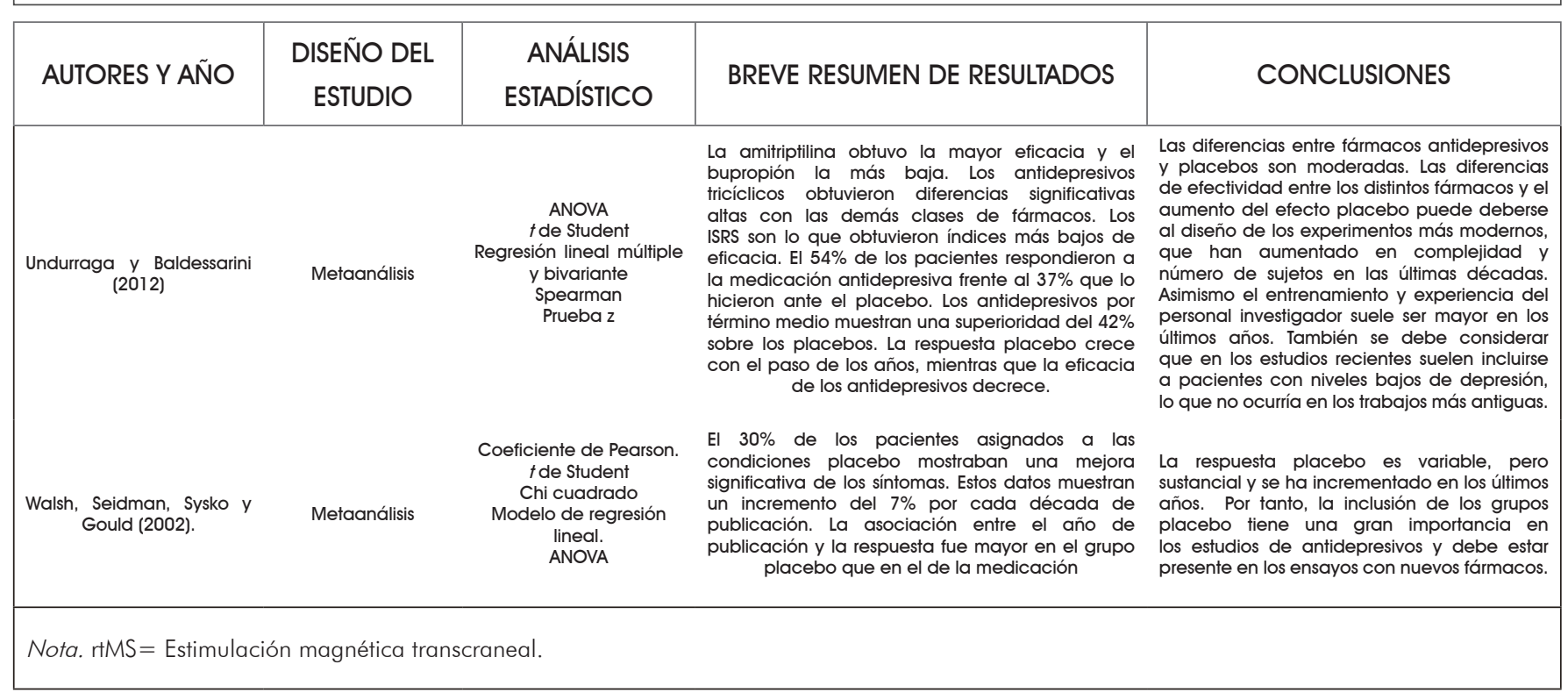

Para facilitar su comprensión, se presentan divididos en tres bloques:

1) se analizará la respuesta placebo a nivel global,

2) se fijará la atención en los factores que la influyen o la modulan y

3) se expondrán los hallazgos encontrados a nivel psicobiológico.

\section{- COMPARACIÓN DE FÁRMACOS ANTIDEPRESIVOS Y PLACEBOS EN TÉRMINOS GENERALES}

En 12 de los estudios se comparó la respuesta de los sujetos ante los antidepresivos y los placebos, destacando que en todos ellos se halló un mayor porcentaje de sujetos que respondían al tratamiento dentro del grupo de la medicación que en el grupo placebo. En cuanto a la eficacia, los antidepresivos se mostraron más eficaces que los placebos en todos los casos, aunque las diferencias entre ellos no fueron siempre significativas.

Los estudios que informan del porcentaje de sujetos que respondieron dentro del grupo de la medicación y los que lo hicieron dentro del grupo del placebo arrojaron los siguientes resultados: en el caso del metaanálisis de Undurraga y Baldessarini (2012) el 54.1\% de los sujetos respondieron a la medicación y el $37 \%$ al placebo. Estos datos son similares a los hallados en varios metaanálisis anteriores, tales como el de Walsh, Seidman, Sysko y Gould (2002) en el que el $50.1 \%$ respondían a la medicación frente al 30\% que lo hacía ante el placebo; el de Kirsh et al. (2008), en el cual el 59\% respondían a la medicación y el $46 \%$ ante el placebo; el de Bridge, Birmaher, lyengar, Barbe y Brent (2009), donde el porcentaje que respondió ante la medicación fue del 59\%, mientras el que lo hizo ante el placebo fue del $46 \%$ o el de Rutherford, Sneed y Roosse (2009), donde el porcentaje de sujetos que respondió a la medicación fue de un 52\%, mientras que el que respondió al placebo fue de un $34.7 \%$. Estos datos también guardan bastante semejanza con los porcentajes obtenidos en la investigación de Lechter, Cook, Witte, Morgan y Abrams (2002) donde el 52\% de los sujetos respondieron a los antidepresivos y $38 \%$ al placebo. Por otra parte, estos autores también obtuvieron diferencias significativas entre los que respondieron ya fuera al placebo o a la medicación frente los que no respondieron a ningún tratamiento $\left(F_{1,49}=121.4, p<.0001\right)$.

En cuanto a la eficacia de los antidepresivos frente a los placebos se encontraron los siguientes resultados: en el metaanálisis realizado por Moncrieff, Wessely y Hardy (2009) se comparaba la eficacia de los antidepresivos tricíclicos vs. placebos activos obteniéndose superioridad de los fármacos $\left(x^{2}(1)=36.3, p<.001\right)$. Por otra parte, Undurraga y Baldessarini (2012) 
encontraron que la respuesta total de los antidepresivos fue de 1.42 (95\% Cl: 1.38-1.48; $z=16.3, p<.0001)$, indicando una superioridad del $42 \%$ de éstos sobre los placebos. Naudet, Millet, Charlier, Reymann, Maria y Falissard (2013) también hallaron resultados similares mostrando el grupo de medicación mejores resultados que el grupo placebo $\left(x^{2}(1)=45.8\right.$, $p<.001)$.

Russowsky, Lopes, Kaptchuk y Fregni (2009) evidenciaron que la respuesta placebo fue elevada en todos los grupos placebos, tanto en los que se comparaban con escitalopram $(d=1.48 ; 95 \%$ C.I. 1.26 a 1.6$)$ como en los que medían la eficacia de la estimulación magnética transcraneal $(d=.82 ; 95 \% \mathrm{Cl}$ : 0.63 1). No obstante, no superaron a los efectos de la medicación.

Para comparar la reducción de la sintomatología depresiva en los sujetos depresivos muchos estudios tomaron como referencia la HRSD. Mancini, Wade, Perugi, Lenox-Smith y Schacht (2014) hallaron que el tamaño del efecto global (ES) entre la duloxetina y el placebo fue de -.272; mientras que Leuchter, Hunter, Tartter y Cook (2014) encontraron que los cambios en la puntuación de la HRSD fueron a nivel numérico, pero no significativos, a favor del grupo de la medicación $\left(x^{2}(1)=3,12, p=.02\right)$. Por otra parte, Kirsch et al. (2008) calcularon que la media de mejora en el grupo con antidepresivos fue de 9.60 puntos en la HRSD, frente a los 7.80 puntos en el grupo placebo, dando como resultado una diferencia de 1.80 a favor de los antidepresivos ( $d=1.24$ para los fármacos y $d=0.92$ para el placebo), siendo la diferencia no significativa.

En la misma línea, Leuchter, Hunter, Tartter y Cook (2014) también informaron de una diferencia numérica, aunque no significativa, a favor del grupo con antidepresivos versus el grupo placebo $\left(x^{2}(1)=3.12, p=.08\right)$, coincidiendo estos resultados también con los de Maybert et al. (2002) y Leuchter, Cook, Witte, Morgan y Abrams (2002), en los que tampoco hubo diferencias significativas en las puntuaciones de las escalas entre los grupos, decreciendo significativamente en la misma cantidad.

\section{- FACTORES QUE INFLUYEN O MODULAN LA RESPUESTA PLACEBO}

En un intento por entender la respuesta placebo, en diversos estudios se han examinado la implicación de diferentes factores.

\section{SEVERIDAD INICIAL}

La severidad inicial de la depresión se perfila como uno de los factores claves en la respuesta placebo, concretamente parece guardar una relación inversa con ella. Tres metaanálisis analizan cómo afecta la severidad a dicha respuesta.

Kirsch et al. (2008), al considerar la severidad del trastorno y comparar la respuesta placebo y la antidepresiva, obtuvieron claros resultados significativos $(d=.50 ; p<.001)$. En concreto, la cantidad de mejora dependía de la línea base de la severidad y era significativa cuando los sujetos obtenían puntuaciones iniciales en HRSD mayores de 28 puntos. Por otro lado, la severidad inicial también presentaba una relación con el tamaño de la muestra $(p=.0586)$, teniendo más poder predictivo en los estudios que incluían muestras pequeñas. Resultados similares fueron encontrados por Bridge, Birmaher, lyengar, Barbe y Brent (2009). Estos autores, tomando la puntuación de la escala CGI como referencia, descubrieron la existencia de una relación inversa entre la respuesta placebo y la severidad del trastorno, siendo la proporción de pacientes con depresión severa que respondieron a la medicación $(r=-$ $.11, p=.78)$ y al placebo $(r=-.69, p=.04)$. Por lo que la severidad de la depresión fue significativamente inversa a la respuesta placebo, aunque la fuerza de la relación fue disminuyendo. Es decir, se producía más placebo cuanto menos grave era la depresión. Sin embargo, la severidad parece no modular en igual medida la respuesta a los antidepresivos. Por último, Russowsky, Lopes, Kaptchuck y Fregni (2009) observaron que los sujetos con depresión severa y refractaria a tratamiento presentaron una baja respuesta al placebo, mostrando una relación inversa $(\beta=-.69, \beta<.001)$.

\section{SESIONES DE SEGUIMIENTO}

Este aspecto había pasado bastante desapercibido por los investigadores, hasta que Posternak y Zimmernan (2007) realizaron un metaanálisis estudiando cómo podían afectar las sesiones de seguimiento a la respuesta placebo. 
Hallaron los siguientes resultados: una sesión extra de seguimiento a las tres semanas estuvo asociada a una reducción de 0.86 en HRSD, mientras que si se producía en la semana $5^{a}$ el descenso era de 0.67. Estos efectos representan del 34-44\% de la respuesta placebo que ocurría en ese marco temporal. Además, dos sesiones más de seguimiento se asociaron al doble de reducción que si se producía solo una, - 0.97 y -1.12 en HRSD, respectivamente.

\section{AÑo DE PUBUCACIÓN}

Un factor que parece estar muy relacionado con el aumento de la respuesta placebo es el año de publicación de los estudios incluidos en los metaanálisis, aspecto que es contemplado en tres metaanálisis. Walsh, Seidman, Sysko y Gould (2002) pusieron de manifiesto que la respuesta placebo se incrementaba significativamente con el año de publicación ( $r=.43 ; 95 \% \mathrm{Cl}$ : 0.18-0.63; $p=.001$ ). Estos datos muestran un incremento del $7 \%$ por cada década de publicación. Al realizar un análisis de regresión lineal, sólo el año de publicación obtuvo datos significativos ( $\beta=.366, t=2.7, p=.009)$. Por su parte, Moncrieff, Wessely y Hardy (2004) hallaron que la respuesta placebo aumentaba con el paso de los años, mientras que la respuesta ante los antidepresivos decrecía. Además, la duración de los ensayos se incrementa a lo largo de los años $(r=.603, p<.0001)$, y en los ensayos que duran más, la respuesta placebo aumentaba en una mayor proporción (pendiente $=1.90$ (95 \% Cl: 0.85-2.95, $p=.005$ ) que la de los fármacos (pendiente $=.92 \quad(95 \% \quad \mathrm{Cl}$ : 0.15 1.99). Más recientemente, Bridge, Birmaher, lyengar, Barbe y Brent (2009), encontraron que la proporción de pacientes que respondieron al placebo correlacionaba significativamente con el año de publicación, indicando un incremento de respuesta con el paso de los años $(\mathrm{N}=10$; $r=.64$; 95\% Cl: 0.02-0.91, $p=.05)$.

\section{EXPECTATIVAS}

A pesar de ser un constructo ampliamente relacionado con la aparición de la respuesta placebo sólo dos estudios evalúan las expectativas en sus análisis. Leutcher Hunter, Tartter y Cook (2014) encontraron una relación significativa entre las expectativas ante la medicación y el porcentaje de cambio en HRSD en el grupo placebo $(\beta=-.17, p<.01)$. Por tanto, unas elevadas expectativas de eficacia predijeron un gran descenso de los síntomas por encima de la severidad del trastorno $(\beta=.16$, $p<.05$ ). Sin embargo, en el grupo que tomaba antidepresivos no se estableció esta relación ( $\beta=-.01, p=.82$ ). Por su parte, Rutherford, Wall, Glass y Stewart (2015) descubrieron que las expectativas de los pacientes influyen en que los síntomas aumenten o disminuyan inmediatamente después del cambio de tratamiento y el conocimiento de su situación experimental tanto en el grupo placebo $(r=$ $.48, p<.001$ ) como en el que sigue tomando la medicación $(r=-.46, p<.001)$.

\section{APOYO TERAPÉUTICO}

Fue evaluado en el estudio de Leutcher, Hunter, Tartter y Cook (2014), arrojando interesantes resultados; concretamente, se encontraron diferencias significativas entre los grupos que recibían apoyo terapéutico junto a la condición placebo o medicación frente a los que sólo recibían apoyo aisladamente $\left(x^{2}(1)=\right.$ $20.85, p<.0001$ y $\left.x^{2}(1)=37.39, p<.0001\right)$. Además, los sujetos que solo recibían apoyo terapéutico fueron más discontinuos a la hora de seguir el tratamiento $\left(x^{2}(1)=4.40, p<.05\right)$ y permanecieron en él menos tiempo que los que tomaban pastillas $(t=-2.97, p<.01)$. Otro aspecto a destacar es que estos pacientes partieron con unas expectativas de la eficacia del tratamiento más baja (media $=3.17,95 \%$ $\mathrm{Cl}$ : 2.76-0.57) comparados con los que fueron asignados a la condición placebo (media $=3.94,95 \% \mathrm{Cl}: 3.56-4.22$ ).

\section{RECUPERACIÓN ESPONTÁNEA}

El metaanálisis de Rutherford, Mori, Sneed, Pimontel y Rose (2012) estudió la contribución de la recuperación espontánea al efecto placebo, encontrando que el tamaño del efecto durante la lista de espera fue .050 (95\% Cl: 0.271-0.739, $p<.001$ ), lo que suponía una media de mejora de 4 puntos en la HRSD. Ésta representa el 40\% de la mejora que se observa con la administración de placebo. 


\section{EDAD}

La edad fue examinada en casi todo los estudios, no obteniéndose resultados significativos; sin embargo, en el metaanálisis de Bridge, Birmaher, lyengar, Barbe y Brent (2009), que analizaba la respuesta placebo en la infancia y la adolescencia, se observó que el porcentaje de respuesta en el grupo placebo en los niños fue del $54.3 \%$, frente al $44.9 \%$ en el grupo de adolescentes $\left(x^{2}=5.87, p=.02\right)$. Por lo tanto, los más jóvenes mostraban una respuesta placebo superior, aunque no significativa.

\section{AUANZA TERAPÉUTICA}

En concreto, Leutcher Hunter, Tartter y Cook (2014) constataron que el subcomponente compromiso era importante $\left(t_{69}=-2.35, p<.05\right)$.

\section{OTROS FACTORES}

Aparte de los factores descritos con anterioridad, los estudios analizados también contemplaron otros diferentes, no hallando resultados significativos destacables pero sí la existencia de algún tipo de relación con la respuesta placebo. Por ejemplo, Rutherford, Sneed y Roosse (2009) examinaron cómo el tipo de diseño del estudio podía influir en el efecto placebo; en concreto, compararon la influencia de dos tipos de grupos controles, aquellos que utilizaban un placebo o un "comparator trial", concluyendo que en el grupo placebo tanto el porcentaje de respuesta (34.7\%) como de remisión (24.5\%) eran inferiores a los que se producían en los grupos "comparator trial", de $65.2 \%$ y $48.4 \%$ respectivamente.

El tipo de tratamiento que incluye grupos placebo como grupo control también ha sido analizado; así, Russowsky, Lopes, Kaptchuck y Fregni (2009) midieron la respuesta placebo en los estudios farmacológicos $(d=1.48 ; 95 \%$ $\mathrm{Cl}: 1.26-1.6)$ y en los estudios donde se utiliza la estimulación magnética transcraneal (rTMS) $(d=0.82 ; 95 \% \mathrm{Cl}: 0.63-1)$, hallando más efecto placebo en los primeros. Puede que el hecho de tomar una pastilla forme parte de un ritual médico más asentado y sobre el que los pacientes tengan más expectativas de mejora que con la rTMS. Los pacientes que presentaban depresión refractaria no parecen responder a ningún tipo de tratamiento ya sea placebo, farmacológico o rTMS.

Finalmente, Mancini, Wade, Perugi, LenoxSmith y Schacht (2014), aunque no hallaron interacciones estadísticamente significativas, encontraron que muchos de los factores presentados anteriormente afectaban al tamaño global del efecto: la leve sintomatología inicial de la depresión y un número de evaluaciones de seguimiento mayor afectaban positivamente a la respuesta placebo. También consideraron que la corta duración de los experimentos y una no adecuada aleatorización podían influir positivamente en ella.

\section{- PSICOBIOLOGÍA DE LA RESPUESTA PLACEBO EN LA DEPRESIÓN}

Cuatro estudios se centraron en dilucidar los mecanismos psicobiológicos asociados a la respuesta placebo. Mayberg et al. (2002) se centraron en los aspectos neuroquímicos de la respuesta placebo, observando un incremento en el metabolismo de la glucosa en las siguientes zonas cerebrales: corteza prefrontal, corteza premotora, corteza parietal inferior, ínsula posterior y área cingulada posterior en el grupo placebo $\left(F_{19,72}=3.97, p<.0001\right)$, mientras que se producía una disminución en el metabolismo en área subgenual, hipotálamo, tálamo, ínsula suplementaria sensorial y parahipocampo. Por otra parte, Leuchter, Cook, Witte, Morgan y Abrams (2002) examinaron la actividad cerebral en el grupo placebo, apreciando un incremento de la actividad prefrontal a partir de la $4^{a}$ semana de tratamiento, que se convirtió en estadísticamente significativo en la $8^{a}$ semana $\left(F_{3,43}=10.11, p<.00004\right)$. En cambio, en el grupo de sujetos que respondía a la medicación antidepresiva se produjo un descenso de la actividad prefrontal, sobre todo en la $2^{a}$ semana al compararlo con los otros grupos $\left(F_{3,45}=8.50\right.$, $p<.0001)$.

Peciña et al. (2015), recientemente, también analizaron los mecanismos neuroquímicos y exploraron las diferencias entre el placebo "activo", el placebo "inactivo" y los antidepresivos. En primer lugar, observaron una activación del receptor $\mu$-opioide en diversas áreas cerebrales (tálamo, corteza cingulada, núcleo accumbens y amígdala) tras la administración del placebo $\left(N=35 ; R^{2}=.32\right.$; 
$p<.001)$. La activación del núcleo accumbens estaba igualmente asociada a una mejora de la respuesta al tratamiento antidepresivo $(\mathrm{N}=35$; $R^{2}=.22 ; p=.01$ ). Además, el placebo "activo" se diferenció del placebo "inactivo" tras la primera semana $(\beta=5, p=.03)$, y los sujetos que recibieron el placebo "activo" en primer lugar obtuvieron diferencias mayores que los que lo recibieron en segundo lugar $(t=-2.1 ; p=.04)$.

Sikora et al. (2016) investigaron si existía una relación entre la conectividad funcional en estado de reposo y los efectos de los antidepresivos y el placebo, hallando que las reducciones de los síntomas depresivos después de una semana con el placebo "activo" fueron superiores que con el "inactivo" ( $F=7.2, p<.012)$. Los sujetos que tenían una elevada conectividad funcional en estado de reposo en la corteza cingulada anterior, previa al inicio del experimento, desarrollaban una marcada respuesta al placebo $(Z=3.97 ; p<.05)$. Además, el placebo inducía reducciones de esta actividad, en concreto, de la activación de la "red de saliencia" en la corteza cingulada anterior $(Z=4.35)$, que se relaciona con la disminución de los síntomas depresivos; esta conectividad podría explicar el $65 \%$ de la varianza atribuida al placebo $\left(R^{2}=.65\right)$. Esta relación también se observa tras la toma de fármacos, aunque después de 10 semanas de tratamiento $(Z=3.09 ; p<.05)$. Por tanto, estos dos últimos estudios coinciden en la superioridad de los placebos "activos" sobre los "inactivos".

\section{DISCUSIÓN}

\section{- RESUMEN DE LOS RESULTADOS}

Esta revisión sistemática se centra en analizar los artículos publicados en los que se recogen investigaciones que estudian la respuesta placebo en la depresión. Diecisiete estudios cumplían los criterios de inclusión exigidos. La respuesta placebo en la depresión es un fenómeno complejo para la que se requiere todavía realizar un mayor número de investigaciones con el fin de intentar establecer los mecanismos biopsicosociales responsables de su aparición. No obstante, ya se empieza a comprender la implicación de algunos factores y los cambios que se producen a nivel cerebral.

\section{- INTERPRETACIONES, VALORACIONES Y CONCLUSIONES DERIVADAS DE LOS RESULTADOS}

Los antidepresivos fueron más eficaces que los placebos en todos los estudios, aunque las diferencias entre ellos no fueran siempre significativas. Asimismo, cabe destacar que en todos ellos se observó un mayor porcentaje de sujetos que respondían al tratamiento dentro el grupo de la medicación que en el grupo placebo (Undurraga \& Baldessarini, 2012; Walsh, Seidman, Sysko \& Gould, 2002; Kirsh et al., 2008; Bridge, Birmaher, lyengar, Barbe \& Brent, 2009; Rutherford, Sneed \& Roosse, 2009; Lechter, Cook, Witte, Morgan \& Abrams, 2002; Moncrieff, Wessely \& Hardy, 2009; Russowsky, Lopes, Kaptchuk \& Fregni, 2009; Mancini, Wade, Perugi, Lenox-Smit \& Schacht, 2014; Leuchter, Hunter, Tartter \& Cook, 2014; Maybert et. al 2002; Naudet et al., 2013). No obstante, algunos autores como Moncrieff, Wessely y Hardy (2009) y Kirsch et al. (2008) consideran que los efectos de los antidepresivos pueden estar sobreestimados y los de los placebos subestimados. Por otro lado, los resultados obtenidos indican que la severidad inicial de la depresión se presenta como un factor crucial para poder entender la respuesta placebo. Los autores coinciden al señalar la existencia de una relación inversa significativa entre la respuesta placebo y la severidad de la depresión, es decir, se produce más respuesta placebo cuanto menos grave es la depresión y viceversa (Kirsh et al., 2008; Bridge, Birmaher, lyengar, Barbe \& Brent, 2009; Russowsky, Lopes, Kaptchuck \& Fregni, 2009). Además, ésta diferencia es atribuida a una disminución de la respuesta ante el placebo y no a un incremento de respuesta ante la medicación (Kirsh et al., 2008).

El número de sesiones de seguimiento también parece provocar un impacto terapéutico positivo sobre la respuesta placebo, representando hasta el $40 \%$ de dicha respuesta (Posternak \& Zimmernan 2007; Mancini, Wade, Perugi, Lenox-Smith \& Schacht, 2014), por lo que se sugiere que el impacto terapéutico es acumulativo y proporcional. Este hecho indica que la respuesta placebo depende en gran medida de la atención y el cuidado recibido durante el curso de los ensayos clínicos. 
Otros constructos sobre los que se sustenta la respuesta placebo son las expectativas y la alianza terapéutica. Unas elevadas expectativas predijeron un gran descenso de los síntomas por encima de la severidad del trastorno (Leutcher Hunter, Tartter \& Cook, 2014). Además, las expectativas influyeron desde el inicio del tratamiento y produjeron variaciones ante los cambios experimentales que se producían a lo largo del mismo, incluso la simple posibilidad de recibir placebo fue asociada a un empeoramiento de los síntomas en los estudios de Rutherford, Wall, Glass y Stewart (2015). Por otra parte, la alianza terapéutica es un fuerte predictor de la respuesta placebo, demostrando que desde las etapas iniciales del tratamiento se establecen con los investigadores relaciones intensas que tendrán su reflejo en la respuesta de los sujetos (Leutcher Hunter, Tartter \& Cook, 2014).

Por otra parte, el estudio de Leutcher Hunter, Tartter y Cook (2014) es el único en el que se mide el "apoyo terapéutico" de manera aislada y se compara con el placebo. Quizás el que se obtengan pobres resultados a favor del apoyo terapéutico puede indicar cómo los sujetos atribuyen más expectativas de mejora a las pastillas, aunque sean placebos, que a la psicoterapia, en línea con lo arraigado que se encuentra en nuestra sociedad el modelo biomédico.

En cuanto a la edad, fue analizada en casi todo los estudios, sin resultados significativos; sin embargo, Bridge, Birmaher, lyengar, Barbe y Brent (2009), al analizar la respuesta placebo en la infancia y la adolescencia, sí apreciaron cierta relación entre una menor edad de los sujetos y una mayor respuesta placebo. Tal vez en esta franja de población la respuesta placebo muestra ciertas peculiaridades.

Otro factor a tener en cuenta es la recuperación espontánea, como se aprecia en el estudio de Rutherford, Mori, Sneed, Pimontel y Rose (2012), donde los pacientes mejoraron de forma sustancial incluso sin tratamiento; de este modo, la recuperación espontánea representaba una mejora aproximada del $40 \%$ distinta a la observada en la mayoría de los estudios sobre la respuesta placebo. Las causas de esta mejoría son desconocidas.

Al analizar la psicobiología de la respuesta placebo se confirma la hipótesis de que se asocia a cambios en la función cerebral. Mayberg et al. (2002) concluyeron que el placebo produce un incremento del metabolismo de la glucosa en algunas áreas cerebrales, sin embargo, la respuesta a la medicación, además de presentar estos cambios en mayor intensidad, mostraba otros específicos y únicos en el núcleo estriado, el hipocampo y el tronco encefálico. Además, los resultados de este estudio sugieren que la adaptación recíproca entre los sistemas límbico y cortical es necesaria para reducir la depresión, independientemente del tipo de tratamiento seguido. No obstante, Leuchter, Cook, Witte, Morgan y Abrams (2002) plantean resultados discrepantes. Estos autores también consideran que el placebo induce cambios en la función cerebral, pero afirman que son distintos a los causados por la medicación antidepresiva, provocando la respuesta placebo altos niveles de actividad metabólica, y presentando un patrón único en el área prefrontal. Por tanto, este estudio sugiere que la respuesta placebo y la respuesta antidepresiva podrían estar asociadas a mecanismos de acción diferentes.

Peciña et al. (2015), por su parte, observaron que el placebo inducía la activación del sistema $\mu$-opioide en el núcleo accumbens, que a su vez está implicado en el desarrollo de la respuesta antidepresiva, así como en la respuesta de resiliencia a la enfermedad. Se cree que el sistema opioide podría actuar modulando la sintomatología depresiva. Esta activación también aparece en otras situaciones como el "dolor", sugiriendo que existe un mecanismo neurobiológico del placebo común en distintas patologías. Recientemente, Sikora et al. (2016), en un novedoso estudio sobre la conectividad funcional en estado de reposo y los efectos de los antidepresivos y el placebo, encontraron que el placebo inducía reducciones de esta actividad en la corteza cingulada anterior, y este hecho se relaciona con la disminución de los síntomas depresivos.

Por último, un curioso factor que parece estar muy relacionado con el aumento de la respuesta placebo es el año de publicación de los trabajos. Los autores atribuyen este hecho a que cada vez participan en los estudios más pacientes con formas leves y moderadas de depresión y la forma de seleccionar a los sujetos ha cambiado, al igual que el diseño de los experimentos (Walsh, Seidman, Sysko \& Gould, 2002; Moncrieff, Wessely \& Hardy, 2004; Bridge, Birmaher, lyengar, Barbe \& Brent, 2009). 


\section{- IMPLICACIONES DE LOS RESULTADOS Y} FUTURAS LIINEAS DE INVESTIGACIÓN

Conocer cómo se produce la respuesta placebo puede ayudarnos a entender los mecanismos biológicos, psicológicos y sociales que influyen en la recuperación de la depresión, siendo también muy importante para optimizar los estudios que examinan la eficacia de los antidepresivos. Además, estos hallazgos extraídos de la revisión pueden ser útiles para valorar la necesidad de someter a los pacientes a tratamiento farmacológico. Este tema de investigación se encuentra todavía en sus inicios, por lo que resulta necesario realizar estudios adicionales que amplíen e intenten replicar los hallazgos encontrados hasta ahora. Asimismo, sería interesante que en futuros estudios los grupos que toman la medicación o el placebo no estuviesen al mismo tiempo bajo ningún tipo de terapia psicológica adicional, puesto que en muchas ocasiones los sujetos que participan en los estudios están recibiendo a la vez alguna terapia, la cual no se tiene en cuenta a la hora de analizar los resultados, siendo la mejoría que se deriva de ella atribuida al fármaco o al placebo. Igualmente, los escasos trabajos en los que se describe algún tipo de "apoyo terapéutico" suele consistir en sesiones de seguimiento llevadas a cabo por personal de enfermería y no en terapias eficaces conducidas por psicólogos.

Del mismo modo, es fundamental profundizar en el conocimiento de las expectativas. En primer lugar, deberían desarrollarse más estudios sobre el rol que juegan en las etapas iniciales. Por ejemplo, en el estudio de Leutcher Hunter, Tartter y Cook (2014), los participantes tenían preferencia por los fármacos, pero sería interesante analizar qué pasaría en sujetos cuyas actitudes y expectativas fueran diferentes. Además, esta variable puede ser influenciada por la personalidad, las actitudes hacia la salud, el sistema de creencias, los factores sociales o las experiencias, y en la actualidad no existen estudios que midan este tipo de factores adicionales que pueden estar modulándolas en un sentido u otro.

Respecto a los aspectos psicobiológicos, se están empezando a investigar y todavía queda un largo camino. Sería importante establecer si los mecanismos de acción del efecto placebo son iguales a los de la depresión o, como postulan algunos autores, son diferentes. De igual forma, sería interesante estudiar cómo afectan las variantes genéticas (polimorfismos) a la respuesta placebo a nivel cerebral.

\section{- $\quad$ LIMITACIONES DE LA REVISIÓN Y POTENCIALES SESGOS DE LOS ESTUDIOS ORIGINALES}

Nuestra revisión sistemática no está exenta de limitaciones, pues aunque se ha pretendido abarcar todo los estudios existentes y publicados acerca de la respuesta placebo en la depresión desde el año 2000, ciertamente se ha podido obviar algún trabajo. Otra posible limitación sería que los estudios incluidos en la revisión presentan algunos problemas; por ejemplo, en el meta-análisis de Bridge, Birmaher, lyengar, Barbe y Brent (2009) no se examinaron las interacciones entre los diferentes factores. También existe una limitación para generalizar los resultados debido a que las características de la población son específicas y los instrumentos de medida arrojan diferentes resultados, en ocasiones midiendo diferentes aspectos.

Otro aspecto a destacar es que la mayoría de los estudios analizados fijaban su atención en 6-8 o 10 semanas de tratamiento, pero no tenemos suficiente información sobre la respuesta placebo y la respuesta antidepresiva a largo plazo. Otra limitación con la que cuentan algunos metaanálisis es que algunos pacientes recibían terapia cognitiva-conductual conjuntamente con los medicamentos o el placebo y este hecho no se controló ni se midió de ningún modo. Al igual que tampoco se controlaron otros factores importantes como los rasgos de personalidad.

\section{- CONSISTENCIA DE LOS HALLAZGOS Y APLICABILIDAD}

En conclusión, podemos recalcar que los resultados obtenidos en la presente revisión coinciden con los encontrados en anteriores revisiones y publicaciones (Benedetti, 2014); por lo tanto, podemos afirmar que los datos se consolidan y la respuesta placebo es real y cuenta con un gran potencial terapéutico. Esto nos puede plantear cierto dilema ético: si el efecto terapéutico del placebo es muy similar al de los antidepresivos, sería muy tentador utilizar 
placebo en lugar de antidepresivos en algunas situaciones, como es el caso de las depresiones moderadas o leves, soslayando así la aparición de los efectos secundarios asociados a los fármacos. En este sentido, se están desarrollando algunos estudios en el que se parte de la hipótesis de que los placebos pueden ser efectivos aun cuando el paciente sabe que está tomando placebo (Kaptchuk et al., 2010). Sin embargo, esta línea de investigación requiere todavía un mayor apoyo empírico para que pueda aplicarse en la práctica clínica.

\section{- Conflicto de intereses}

Los autores declaran no tener ningún conflicto de intereses.

\section{REFERENCIAS}

*Artículos incluidos en la revisión sistemática.

Aguilar, E. (2006). Neurobiología de la respuesta placebo. Revista Mexicana de Neurociencia, 7, 300-307.

Amanzio, M. \& Benedetti, F. (1999). Neuropharmacological dissection of placebo analgesia: expectation activated opioid systems versus conditioning-activated specific subsystems. Journal of Neuroscience, 19, 484-494.

Andrews, P. W., Thomson, J. A., Amstadter A. \& Neale M.

C. (2012). Primum non nocere: an evolutionary analysis of whether antidepressants do more harm than good. Frontiers in Psychology, 3 (117), 1 - 17.

Bandura, A. (1977). Self-efficacy: toward a unifying theory of behavior change. Psychological Review, 84, 191215.

Benedetti, F. (1996). The opposite effects of the opiate antagonist naloxone and the cholecystokinin antagonist proglumide on placebo analgesia. Pain, 64, 535-543.

Benedetti F. (1997). Cholecystokinin type A and type B receptors and their modulation of opioid analgesia. News Physiological Science, 12, 263-268.

Benedetti F. (2009). Placebo Effects: Understanding the Mechanisms in Health and Disease. Oxford, UK: Oxford University Press.

Benedetii, F. (2014). Placebo effects. New York, US: Oxford University Press.

Benedetti, F., Amanzio, M. \& Maggi, G. (1995). Potentiation of placebo analgesia by proglumide. Lancet, 346, 1231.

Benedetti, F., Pollo, A., Lopiano, L., Lanotte, M., Vighetti, S. \& Rainero I. (2003). Conscious expectation and unconscious conditioning in analgesic, motor and hormonal placebo/nocebo responses. Journal of Neuroscience, 23, 4315-4323.

*Bridge, J. A., Birmaher, B., lyengar, S., Barbe, R.P. \& Brent,
D.A. (2009). Placebo response in randomized controlled trials of antidepressants for pediatric mayor depressive disorder. American Journal of Psychiatry, 166, 42-49.

Brody, H. (2000). The placebo response. New York, US: Harper Collins.

Choliz, M. \& Capafons, A. (1991). Un intento de reconceptualización del fenómeno placebo. Psicothema, 3, 319-336.

Colloca, L. \& Benedetti, F. (2005) Placebos and painkillers: is mind as real as matter? Nature Reviews Neuroscience, 6, 545-552.

De Pascalis, V., Chiaradia, C. \& Carotenuto, E. (2002). The contribution of suggestibility and expectation to placebo analgesia phenomenon in an experimental setting. Pain, 96, 393-402.

Doering, B.K. \& Rief, W. (2012). Utilizing placebo mechanisms for dose reduction in pharmacotherapy. Trends in Pharmacological Sciences, 33, 165-172.

Ferreira-González, I., Urrutia, G. \& Alonso-Coello, P. (2011). Revisiones sistemáticas y metaanálisis: bases conceptuales e interpretación. Revista Española de Cardiología, 64, 688-696.

Finnis, D.G, Kaptchuk, T.J., Miller, F. \& Benedetti, F. (2010). Placebo effects: Biological, Clinical and Ethical Advances. Lancet, 375, 686-695.

Fuente-Fernández, R., Ruth, T.J., Sossi, V., Schulzer, M., Calne, D.B. \& Stoessl, A.J. (2001). Expectation and dopamine release: mechanism of the placebo effect in Parkinson's disease. Science. 293, 1164-1166.

Furmark, T., Appel, L. \& Henningsson, S. (2008). A link between serotin-related gene polymorphisms, amygdala activity, and placebo-induced relief from social anxiety. Journal of Neuroscience, 28, 1366-1374.

Geers, A.L., Helfer, S.G., Kosbab, K., Weiland, P.E. \& Landry, S.J. (2005). Reconsidering the role of personality in placebo responding: an interactionist perspective. Journal of Psychosomatic Research, 58, 121-127.

Geers, A.L., Wellman, J.A., Fowler, S.L., Helfer, S.G. \& France, C.R. (2010). Dispositional optimism predicts placebo analgesia. Journal of Pain, 11, 1165-1171.

Goebel, M.U., Trebst, A.E., Steiner, J., Xie, Y.F., Exton, M.S. \& Frede, S. (2002). Behavioral conditioning of immunosuppression is possible in humans. Federation of American Societies for Experimental Biology Journal, 16, 1869-1873.

Harrington, A. (1997). The placebo Effect: an Interdisciplinary Exploration. Cambridge, MS: Harvard University Press.

Kaptchuk, T. J. (2001). Is the placebo powerless? New England Journal of Medicine, 345, 1277-1282.

Kaptchuk, T. J., Friedlander, E., Kelley, J. M., Sanchez, M. N., Kokkotou, E., Singer, J. P. \& Lembo, A. J. (2010). 
Placebos without deception: A randomized controlled trial in irritable bowel syndrome. Public Library of Science One, 5, e15591.

Kaptchuk, T.J., Kerr, C.E., Zanger, A. (2009). Placebo controls, exorcisms and the devil. Lancet, 374, 1234 1235.

Kessler, R. C., Chiu, W.T. \& Demler, O. (2005). Prevalence, severity, and comorbidity of twelve-month DSMIV disorders in the National Comorbidity Survey Replication. Archives of General Psychiatry, 62, 617 627.

*Kirsch, I., Deacon, B.J., Huedo-Medina, T.B., Scoboria, A., Moore, T.J. \& Johnson, B.T. (2008). Initial severity and antidepressant benefits: a meta-analysis of data submitted to the food and drug administration. Public Library of Science, 5, 260-268.

Kirsch, I. y Sapirstein, G. (1998). Listening to prozac but hearing placebo: a meta-analysis of antidepressant medication. Prevention and Treatment, 1, 1-16.

*Mancini, M., Wade, A.G., Perugi, G., Lenox-Smith, A., Schacht, A. (2014). Impact of patient selection and study characteristics on signal detection in placebocontrolled trial with antidepressants. Journal of Psychiatric Research, 51, 21-29.

*Mayberg, H.S., Silva, J.A., Brannan, S.K., Tekell, J.L., Mahurin, R.K., McGinnis, S. \& Jerabek, P.A. (2002). The functional neuroanatomy of the placebo effect. American Journal of Psychiatry, 159, 728-737.

Moher, D., Shamseer, L., Clarke, M., Ghersi, D., Liberati, A., Petticrew, M., Shekelle, P., \& Stewart, L. (2015). Preferred reporting items for systematic reviews and meta-analyses protocols (PRISMA-P) 2015 statement. Systematic Reviews, 4, 1-9.

*Moncrieff, J., Wessely, S. \& Hardy, R. (2004). Active placebos versus antidepressants for depression. Cochrane Database of Systemic Reviews, 1, CD003012.

Montgomery, G.H. \& Kirsch, I. (1997) Classical conditioning and the placebo effect. Pain, 72, 107 113.

Mora, M.S., Nestorius, Y. \& Rief, W. (2011). Lessons learned from placebo groups in antidepressant trials. Philosophical Transactions of the Royal Society B: Biological Sciences, 366, 1879-1888.

Morton, D.L., Watson, A., El-Deredy, W. \& Jones, A.K. (2009). Reproducibility of placebo analgesia: effect of dispositional optimism. Pain, 146, 194-198.

*Naudet, F., Millet, B., Charlier, P., Reymann, J.M., Maria, A.S. \& Falissard, B. (2013). Which placebo to cure depression? A thought-provoking network metaanalysis. BioMed Central Medicine, 11 , 230-240.

*Leuchter, A.F., Cook, I.A., Witte, E.A., Morgan, M. \&
Abrams, M. (2002). Changes in brain function of depressed subjects during treatment with placebo. American Journal of Psychiatry, 159, 122-129.

*Leuchter, A.F., Hunter, A.M., Tartter, M. \& Cook, A. (2014). Role of pill-taking, expectation and therapeutic alliance in the placebo response in clinical trials for mayor depression. The British Journal of Psychiatry, 205, 443-449.

Lieberman, M.D. (2004). The neural correlates of placebo effects: a disruption account. Neuroimage, 22, 447-455.

*Peciña, M., Bohnert, A.B., Sikora, M., Avery, B.A., Langenecker, S.A., Mickey, B.J. \& Zubieta, J. (2015). Placebo-actived neural systems are linked to antidepressant responses: neurochemistry of placebo effects in major depression. Journal of the American Medical Association, 72, 1087-1094.

*Posternak y Zimmerman (2007). Therapeutic effect of follow-up assessments on antidepressant and placebo response rates in antidepressant efficacy trials. British Journal of Psychiatry, 190, 287-292.

Price, D.D., Finniss, D.G. \& Benedetti, F. (2008). A comprehensive review of the placebo effect: recent advances and current thought. Annual Review of Psychology, 59, 565-590.

*Russowsky, A., Lopes, M., Kaptchuck, T.J. \& Fregni, F. (2009). Placebo response of non-pharmacological trials in mayor depression: a systematic review and meta-analysis. Public Library of Science, 4, e4824.

*Rutherford, B.R., Mori, S., Sneed, J.R., Pimontel, M.A. \& Roose (2012). Contribution of spontaneous improvement to placebo response in depression: a meta-analytic review. Journal of Psychiatry Research, 46, 697-702.

Rutherford, B.R. \& Roose, S.P. (2012). A model of placebo response in antidepressant clinical trials. American Journal of Psychiatry, 170, 723-733.

*Rutherford, B.R., Sneed, J.R. \& Roose, S.P. (2009). Does study design influence outcome? The effects of placebo control and treatment duration in antidepressant trials. Psychotherapy \& Psychosomomatics, 78, 172181.

*Rutherford, B.R., Wall, M.M., Glass, M.S. \& Stewart, J.W. (2015). The role of patient expectancy in placebo and nocebo effects in antidepressant trials. Journal of Clinical Psychiatry, 75, 1040-1046.

Sanchís, J. (2012). El placebo y el efecto placebo. Medicina Respiratoria, 5, 37-46.

*Sikora, M., Heffernan, J., Avery, E.T., Mickey, B.J., Zubieta, J. \& Peciña, M. (2016). Salience network functional connectivity predicts placebo effects in mayor depression. Biological Psychiatry, 1, 68-76. 
Stewart-Williams, S. \& Podd, J. (2004). The placebo effect: dissolving the expectancy versus conditioning debate. Psychological Bulletin, 130, 324-340.

*Undurraga, J. \& Baldessarini, R.J. (2012). Randomized, placebo-controlled trials of antidepressants for acute mayor depression: thirty-year meta-analytic review. Neuropsychopharmacology, 37, 851-864.

Wager, T.D. (2004). Placebo-induced changes in FMRI in the anticipation and experience of pain. Science, 303, $1162-1167$.
Wager, T.D. (2005). The neural basis of placebo effects in anticipation and pain. Seminar in Pain Medicine, 3, 22-30.

*Walsh, B.T., Seidman, S., Sysko, R. \& Gould, M. (2002). Placebo response in studies of major depression. Variable, substantial and growing. The Journal of the American Medical Association, 287, 1840-1847. 